\title{
EMPREENDEDORISMO CULTURAL E ARTÍSTICO: VEREDAS DA PESQUISA ACADÊMICA
}

CULTURAL AND ARTISTIC ENTREPRENEURSHIP: PAHTS FOR ACADEMIC RESEARCH

Recebido em 22.09.2020 provado em 22.12.2020

Avaliado pelo sistema double blind review

DOI: https://doi.org/10.12712/rpca.v14i4.46268

\section{Simony Rodrigues Marins}

simonymarins@gmail.com

Núcleo de Pós-Graduação em Administração. Escola de Administração/Universidade Federal da Bahia -

Salvador/Bahia, Brasil

$\underline{0000-0003-3881-1194}$

\section{Eduardo Paes Barreto Davel}

davel.eduardo@gmail.com

Núcleo de Pós-Graduação em Administração. Escola de Administração/Universidade Federal da Bahia -

Salvador/Bahia, Brasil

$\underline{0000-0003-0610-6474}$

\section{Resumo}

A produção acadêmica sobre o empreendedorismo nas artes e cultura é dispersa e as pesquisas conceituais-teóricas integrativas são limitadas. O objetivo deste artigo é consolidar uma compreensão integrada da produção acadêmica sobre a relação entre empreendedorismo, cultura e arte e propor perspectivas renovadoras para pesquisas futuras. Os resultados categorizam singularidades (valor, inovação e consumo subjetivos, lógicas artística, cultural, paradoxal e arriscada), temáticas, impactos (econômicos, sociais, culturais, artísticos e políticos, diretos, indiretos, positivos e negativos), forças e desafios. A estética organizacional é proposta e discutida como perspectiva teórico-conceitual para renovar pesquisas futuras.

Palavras-chave: Empreendedorismo. Empreendedorismo cultural. Empreendedorismo artístico. Estética organizacional.

\begin{abstract}
The academic production on entrepreneurship in the arts and culture is dispersed and the integrative conceptual-theoretical research are limited. The paper goal is to consolidate an integrated understanding of academic production on the relationship between entrepreneurship, culture and art and to propose renewing perspectives for future research. The results categorise singularities (subjective value, innovation and consumption, artistic, cultural, paradoxical and risk-oriented logic), themes, impacts (economic, social, cultural, artistic and political, direct, indirect, positive and negative), strengths and challenges. Organizational aesthetics is proposed and discussed as a theoretical-conceptual perspective for further research renewing.
\end{abstract}

Keywords: Entrepreneurship. Cultural entrepreneurship. Artistic entrepreneurship. Organizational aesthetics. 


\section{Introdução}

A relação entre empreendedorismo, cultura e arte torna-se estratégica desde que a cultura passa a ocupar um lugar extremamente vital para o desenvolvimento das sociedades e economias contemporâneas (Jones, Lorenzen \& Sapsed, 2015; Khaire, 2015, 2017). No horizonte das indústrias/economia criativa (Caves, 2000; Cunningham \& Potts, 2015; Davies \& Sigthorsson, 2013; Hartley, Wen \& Li, 2015; Howkins, 2001; Jones, Lorenzen \& Sapsed, 2015; Throsby, 2001; 2010) o empreendedorismo surge favorecido por políticas culturais, principalmente, europeias (Ellmeier, 2003; Naudin, 2015). Neste desenrolar, apesar de sua importância ter crescido (Klamer, 2011; Khaire, 2017, 2015; Loy \& AAgeson; 2018), a compreensão das especificidades do empreendedorismo na cultura, artes e indústrias criativas é ainda uma preocupação recente (Essig, 2017; Hagoort, 2007; Hausmann \& Heinze, 2016; Klamer, 2011) e precariamente consolidada nas pesquisas.

Setores da economia criativa produzem bens com maior valor simbólico que material (Hirsch, 1972), dispondo de elementos de criatividade artística (Caves, 2000). Uma íntima relação entre empreendedorismo e indústrias criativas (Khaire, 2015; 2017; Kolb, 2015; Loy \& AAgeson; 2018; Toghraee \& Monjezi, 2017) se estabelece pela relevância do empreendedorismo na tradução e materialização de capital simbólico e econômico (Townley \& Gulledge, 2015). Esta tradução decorre da criação de trabalho (Ellmeier, 2003), inovação (Cunningham \& Potts, 2015) e mudança econômica, social, cultural, local e global (Jones, Lorenzen, \& Sapsed, 2015), gerando externalidades que ultrapassam a dimensão econômica (Cunningham \& Potts, 2015).

O enfoque nas singularidades do empreendedorismo (Klamer, 2011) nos permite melhor compreender e potencializar o desenvolvimento e a transformação na economia criativa (Jones, Lorenzen \& Sapsed, 2015; Khaire, 2017). Compreender que o valor deriva da criatividade contribui diretamente para a identificação, classificação e diferenciação de produtos e serviços; compreensão tão necessária à acadêmicos e formadores de políticas públicas (Jones, Lorenzen \& Sapsed, 2015). Com isto, buscar melhor entender a relação entre empreendedorismo, arte e cultura se torna vital no contexto da economia criativa.

Apesar de um esforço crescente, o campo de pesquisa sobre empreendedorismo no campo da cultura e das artes encontra-se ainda pouco integrado. Encontramos apenas dois estudos com esse propósito. No primeiro estudo (Davel \& Cora, 2016), o enfoque recaiu na construção de um esquema conceitual sobre empreendedorismo e cultura, a partir de diferentes abordagens (a cultura como discurso, criação e consumo) e processos (mobilização, materialização e consolidação simbólicos). Este estudo desconsidera a produção acadêmica sobre empreendedorismo e arte. $\mathrm{O}$ segundo estudo busca revisar a produção acadêmica sobre empreendedorismo nas indústrias culturais e criativas (Hausmann \& Heinze, 2016) e constata a falta de preocupação com singularidades e educação empreendedora que considerem essas singularidades. Neste estudo, não encontramos novas categorias integradoras e explicativas dessas singularidades. No geral, constatamos um cenário de timidez, polissemias, tensões simbólicas e contradições na produção acadêmica que relaciona empreendedorismo, cultura e arte.

O objetivo deste artigo é consolidar uma compreensão integrada da produção acadêmica sobre a relação entre empreendedorismo, cultura e arte e propor perspectivas renovadoras para pesquisas futuras. A metodologia de pesquisa é baseada em levantamento sistemático e extensivo da produção acadêmica (artigos, livros, capítulos de livros, teses, dissertações) nas principais bases nacionais e internacionais que tratam da relação entre empreendedorismo, arte e cultura. As bases consultadas foram: Spell, Portal Capes, Web of Science, Sage Publications, Emerald Insight, Library of Congress. A busca ocorreu em dois momentos. No primeiro momento, os termos de busca foram: empreendedorismo, cultura e arte. No segundo momento, os termos de busca foram: empreendedorismo artístico, empreendedorismo cultural, empreendedorismo criativo e economia criativa. Em paralelo, à medida que a produção acadêmica era identificada, procedíamos a análise das referências citadas para identificar novas 
referências. Essas referências eram pesquisadas, analisadas e integradas ao repertório desta pesquisa. A análise sistemática do conjunto de pesquisas identificadas permitiu a elaboração e sustentação de categorias centrais para descrever o campo: singularidades (valor, inovação e consumo subjetivos, lógicas artística, cultural, paradoxal e arriscada), temáticas, impactos (econômicos, sociais, culturais, artísticos e políticos, diretos, indiretos, positivos e negativos), forças e desafios. Em seguida, foi realizada uma análise desses resultados de pesquisa para identificar, destacar e propor uma perspectiva que fosse significativa para renovar pesquisas futuras.

A contribuição desta pesquisa é dupla. Ao elaborar uma visão estruturada do empreendedorismo no campo da cultura e da arte, os resultados desta pesquisa sedimentam um patamar de apoio para estimular e melhorar a realização de novas pesquisas. Além disso, os resultados destacam a estética organizacional como perspectiva teórico-conceitual com forte potencial para renovar a produção acadêmica. $\mathrm{O}$ conhecimento gerado nesta pesquisa auxilia educadores de empreendedores em sua prática, bem como empreendedores e formuladores de políticas públicas que atuam no campo da cultura e das artes. Empreendedores podem melhor conhecer e refletir sobre as características singulares e marcantes de sua prática empreendedora. Educadores podem enriquecer o conteúdo de suas práticas de ensinoaprendizagem. Formadores de políticas públicas podem dispor de conhecimentos refinados para melhor estimular o desenvolvimento das sociedades futuras.

\section{Empreendedorismo na cultura e arte: uma análise crítica da produção acadêmica}

A relação direta entre empreendedorismo, cultura e arte é estabelecida na década de 1980, quando um estudo chamou de empreendedor o capitalista cultural bem-sucedido nos negócios, interessado em artes e disposto a assumir responsabilidades no campo da alta cultura (DiMaggio, 1982). No contexto das indústrias criativas, os primeiros teóricos como Caves (2000) e Throsby (2001) não teriam ainda dado ênfase ao empreendedorismo cultural (Klamer, 2011). Isto começou a mudar quando a edição especial de 2003 do Journal of Arts Management, Law and Society, editada por Ruth Rentschler foi publicada com enfoque em questões sobre inovação, criação de valor e microempresas (Essig, 2017). Esta fase é marcada pela crítica sobre os regimes de governança neoliberal (Hesmondhalgh \& Baker, 2011; McRobbie, 2011) como abordagem empreendedora da produção cultural (Ellmeier, 2003; Essig, 2017; McRobbie, 2011). Com o passar do tempo, discussões dentro do campo de estudos culturais sobre cultura de elite e cultura popular, arte verdadeira, entretenimento, massificação, comércio artístico e cultural, economia local e global (Bourdieu, 1993; 1996; Ellmeier, 2003; Hirsch, 1972; Lawrence \& Philips, 2002) passaram a permear as discussões sobre empreendedorismo. Se o termo empreendedorismo é elástico, isto também ocorre dentro das artes e da cultura ao falarmos de setor criativo (Stefanovic, 2018). Há estudos que se referem ao empreendedorismo na economia criativa sem classificá-lo (eg. Jones, Lorenzen \& Sapsed, 2015; Loy \& AAgeson; 2018; McRobbie, 2011), mas de forma geral e intercambiável (Birnkrau, 2018; Stefanovic, 2018) encontramos alguns termos mais recorrentes: empreendedorismo cultural, empreendedorismo artístico e empreendedorismo criativo (Essig, 2017; Hagoort, 2007; Hausmann \& Heinze, 2016).

O conceito de empreendedorismo cultural emergiu como um domínio acadêmico vibrante e fértil nas últimas décadas (Gehman \& Soublière, 2017; Hausmann \& Heinze, 2016; Lounsbury \& Glynn, 2019; Stefanovic, 2018) e as definições apresentam ideias em torno de conceitos como: inovação (Hausmann \& Heinze, 2016; Kavousy et. al, 2010; Scott, 2012; Swedberg, 2006; Toghraee \& Monjezi, 2017; Wilson \& Strokes, 2005), criatividade (Hausman, 2010; Hausmann \& Heinze, 2016; Konrad, 2010; Toghraee \& Monjezi, 2017; Wilson \& Strokes, 2005), exploração de oportunidades (Hausman, 2010; Scott, 2012), riscos e incertezas (Banks et al., 2000; Eikhof \& Haunschild, 2006; 2007; Toghraee \& Monjezi, 2017; Wilson \& Strokes, 2002), mudança e transição (Wilson \& Strokes, 2002, 2005; Toghraee \& Monjezi, 2017), concorrência (Ellmeier, 2003), trabalho (Ellmeier, 2003; Wilson \& Strokes, 2004), criação de empresas (Hausmann, 2010), ação em grandes empresas (Ellmeier, 2003), intraempreendedorismo 
(Toghraee \& Monjezi, 2017; Wilson \& Strokes, 2002), instituições culturais (Kolsteeg, 2013), gerência e gestão (Rentschler, 2007; Toghraee \& Monjezi, 2017; Wilson \& Strokes, 2002; 2004; 2005), financiamento e obtenção de recursos (Toghraee \& Monjezi, 2017; Wilson \& Strokes, 2002; 2005), negócios (Hausmann \& Heinze, 2016), discurso (Kolsteeg, 2013), perfil individual, especial e visionário (Hausmann, \& Heinze, 2016; Toghraee \& Monjezi, 2017; Wilson \& Strokes, 2002; 2004; 2005), contexto (Lange, 2018), desenvolvimento urbano e regional (Hagoort, Thomassen, \& Kooyman, 2012) e responsabilidade social (Hagoort, 2007). Fala-se em combinação e equilíbrio entre valores artístico-simbólicos e econômicos (Davel, \& Cora, 2016; Toghraee \& Monjezi, 2017) ou ainda em economia como suporte ou instrumento para empreender valores culturais e sociais (Banks, Gill \& Taylor, 2014; Eikhof \& Haunschild, 2006; Ellmeier, 2003; Hagoort, 2007; Klamer, 2011; Lange, 2018).

Em 2005, o termo 'empreendedorismo artístico' (Beckman, 2015; Beckman \& Essig, 2012; Chang \& Wyszomirski, 2015; Scherdin \& Zander, 2011; White, 2015; 2017; Preece, 2011; 2014; 2015) é destacado na constituição de um campo que busca distinção em relação ao empreendedorismo cultural (Essig, 2017; Hausmann \& Heinze, 2016). Este termo emerge nas instituições de ensino superior dos EUA (Essig, 2015; 2017; White; 2017). Ao pensarmos o artista como empreendedor (Poorsoltan, 2012), as ideias tratam de gestão, criatividade e autonomia, capacidade de adaptabilidade e criação de valor artístico, econômico e social (Birnkrau, 2018; Chang \& Wyszomirski, 2015), incerteza, talento e carreira (Menger, 2014), inovação (Scherdin \& Zander, 2011; Wennes, 2009; Elias \& Rehn, 2009; White, 2017), valor e expressão artística (Barry, 2011; Beckman, 2015; Beckman \& Essig, 2012; Chang \& Wyszomirski, 2015; Elias et al., 2018; Essig, 2015, 2017; Lindqvist, 2011; Lingo \& Teeper, 2013; Menger, 2014; Meisiek \& Haefliger, 2011; Scherdin \& Zander, 2011; Preece, 2011; Rivetti \& Migliaccio, 2018; White, 2015; 2017).

O empreendedorismo criativo (Bujor \& Avasilcai, 2016; Cora, 2016; Hagoort, Thomassen \& Kooyman, 2012; Hausmann \& Heinze, 2016; Patten, 2016; Rae, 2005; Stefanovic, 2018; Werthes, Mauer \& Brettel, 2017; Svejenova, Slavich \& Sondos, 2015) coloca-se como um conceito geral de abrangência e tendência em torno da criatividade (Stefanovic, 2018). Trata-se de um conceito guarda-chuva localizado no âmbito da economia criativa, mas onde o empreendedor é um criador de valor econômico que deve equilibrar estes objetivos com os valores culturais para produzir desenvolvimento (Hausmann, \& Heinze, 2016), resultados econômicos, criativos e sociais (Stefanovic, 2018). As ideias giram em torno do campo mais amplo da criatividade na economia criativa, incluindo design de computadores, moda e envolvem o investimento em talento por meio de gestão, criação de projetos para gerar valor, ativos intangíveis, como propriedade intelectual, bem-estar, marca, reputação, rede, conhecimento (Stefanovic, 2018) e oportunidades (Rae, 2005; Svejenova, Slavich \& Sondos, 2015).

As diferenças entre esses diversos termos referem-se a questões de pioneirismo e utilização (empreendedorismo cultural como primeiro e mais utilizado), de finalidade e interfaces (arte, cultura e criatividade), de lugar onde são mais promovidos e de foco em educação (artístico: EUA, cultural: Europa, etc.), de vieses mais (cultural) ou menos críticos (criativo)(Naudin, 2015). Entretanto, empreendedorismo artístico e cultural comungam do entendimento que o valor econômico e o lucro não são prioridade ou primordial (Birnkrau, 2018; Essig, 2015; 2017; Hausmann \& Heinze, 2016; Thom, 2015), devendo haver uma busca pelo equilíbrio. Há ainda a percepção de estágios de produção acadêmica (Gehman \& Soublière, 2017; Hagoort, 2007), como as fases de descoberta, conceitual e teórica (Hagoort, 2007), estando o empreendedorismo cultural na fase teórica e o artístico na fase conceitual (Essig, 2017). Há ainda a ideia de fluxos de pesquisa que inserem a atual fase do empreendedorismo cultural como criadora de valor marcada pela performatividade (Gehman \& Soublière, 2017).

\subsection{Singularidades do empreendedorismo na arte e na cultura}

Apesar das distinções, ao tratarmos de empreendedorismo nas artes e na cultura, grande parte das ideias limitam-se à aproximações com as ideias clássicas e padrões do empreendedorismo, o que se torna crítico já que estas ideias tendem a enfatizar a capacidade de empreendedores produzirem lucros financeiros 
(Klamer, 2011; Swedberg, 2006). Embora algumas singularidades sejam apontadas em definições, elas são ainda pouco aprofundadas ou problematizadas. A análise da produção acadêmica em empreendedorismo, cultura e arte nos orientou e permitiu destacar singularidades em relação a obra e ao processo empreendedores.

Valor subjetivo da obra empreendedora. A obra, bens ou serviços artísticos dispõem de valor subjetivo diferenciado (Banks et al., 2000; Elias et al., 2018; Khaire, 2017; Klamer, 2011; Toghraee \& Monjezi, 2017) com relação aos demais bens tradicionais, que é seu significado (Lawrence \& Philips, 2002). Trata-se do simbolismo, do impacto de sensações, sentimentos e percepções de gosto, emoções, identidades, ideias políticas, éticas (Bridgstock, 2012), despertados por exemplo, pela beleza de espetáculos teatrais, fotografias ou músicas. Portanto, estes bens são 'úteis' particularmente pelos fins estéticos (levam a fruição, entretenimento e identificação estético-cultural). Isto diferencia o valor cultural de bens artísticos do valor de bens tradicionais, prioritariamente funcionais (Lawrence \& Philips, 2002; Khaire, 2017). A finalidade estética demarca a importância de valores subjetivos (Hirsch, 1972; Lawrence \& Philips, 2002) e singulariza o empreendedorismo na economia criativa (Toghraee \& Monjezi, 2017).

Por mais que o valor ou a missão esteja no centro de todo o trabalho empreendedor, este valor torna-se central no empreendedorismo nas artes e cultura (Birnkrau, 2018). Isto porque o valor subjetivo cultural, artístico, simbólico, intangível (Banks et al., 2000; Klamer, 2011; Scott, 2012), intrínseco (Bridgstock, 2012; Toghraee \& Monjezi, 2017), estético - é um valor fundamental na realização de objetivos culturais e na produção de mudança cultural (Klamer, 2011; Swedberg, 2006). A mudança ocorre porque o valor transita na sociedade e neste trânsito, o empreendedorismo cultural é o responsável por empreender estes valores (Klamer, 2011) que são inseparáveis de valores estabelecidos no âmbito das sociedades. Com isso, este empreendedorismo precisa, portanto, ser deliberadamente entendido e valorizado (Klamer, 2003).

Valores subjetivos na arte e cultura são críticos à valores de organizações tradicionais, como por exemplo, a simples valorização salarial ou o ideal de criatividade individual para atender ao controle gerencial (Eikhof \& Haunschild, 2006). No teatro, estes valores substituem o modelo hierárquico tradicional na relação entre artistas, diretores e críticos, porque estes valores, na forma de julgamentos de mérito e impacto artístico, geram interpretações que são cooperativas e não instrucionais sobre a qualidade da arte (Eikhof \& Haunschild, 2006). Outros exemplos são o sucesso e fracasso (Formica, 2020; Konrad, 2013; Menger, 2014; Riot, 2013), que são formas de resultados mensurados pela intensidade do gosto, da amizade (Eikhof \& Haunschild, 2006), pela estética, ética, política e religião (Menger, 2014).

Este processo de valorar é distinto porque as artes e a cultura tratam de experiências (Davel, \& Cora, 2016; Lawrence \& Philips, 2002; Ravasi \& Rindova, 2013). Estas experiências são traduzidas na própria obra artística, na identidade cultural específica (Toghraee \& Monjezi, 2017), nas percepções do público consumidor sobre aquele bem ou serviço (Davel \& Cora, 2016; Morris, 2014) ou nos processos inerentes à vida e cotidiano organizacional e artístico (Eikhof \& Haunschild, 2006). Tem-se valores que superam o valor econômico, em bens intangíveis e incomensuráveis (Toghraee \& Monjezi, 2017). Enquanto motivações de artistas ou criadores, os valores subjetivos já foram vistos como poderes raros ou capitais (Becker, 1982), que refletem questões individuais (paixão, devoção, puro prazer, reconhecimento, respeito, autorrealização) e coletivas (necessidades de vínculo, valores de solidariedade) (Eikhof \& Haunschild, 2006), preferências por redes pessoais e locacionais (Konrad, 2013; Preece, 2011). Assim, o valor subjetivo resultante na obra (bens ou serviços) envolve produção, processo criativo e consumo.

Tem-se o envolvimento de bens artísticos em complexos processos de criação de valor (Elias et al., 2018) por transformações de valor incomensurável em materialidade com valor incalculável. Assim, estes bens adquirem valor econômico por processos de construção de sentido que conferem significado interpretável (Khaire, 2015). Esta criação depende da diferença cultural (Frankelius, 2011), já que ela reflete uma co-criação de valor estético com base em imaginação, contemplação e construção de consenso (Elias et al., 2018) e exige a presença de entidades, críticos, comentaristas que também definem 
significado para o público (Becker, 1982; Bourdieu, 1996). Assim, a obra empreendedora envolve processos de narrativas (Khaire, 2015), conscientização e persuasão (Klamer, 2011; Lounsbury \& Glynn, 2019). Por esta natureza complexa de processos, compreender o valor subjetivo solicita diferentes lentes analíticas (Khaire, 2015; Ravasi \& Rindova, 2013). Sociólogos, fenomenólogos da arte e outros, por exemplo, tratam dos valores subjetivos como capitais culturais, sociais e simbólicos (Bourdieu, 2007), humano (Becker, 1964), institucional (Scott, 2012) ou reputacional (Ravasi \& Rindova, 2013). O capital simbólico considera distinções como prestígio, reputação, honra e fama (Bourdieu, 1989) e se coloca como a alternativa dominante de credibilidade passível de conversão em lucro (Bourdieu, 1996).

Consumo subjetivo da obra empreendedora. O consumo artístico é um processo inerentemente experiencial que ocorre na interpretação durante a apreciação da obra enquanto bem (fotografias, pinturas) ou durante a apreciação da obra enquanto serviço performático (música, teatro etc.) (Davel \& Cora, 2016; Lawrence \& Phillips, 2002). Enquanto experiência, há transformação subjetiva do expectador (Davel \& Cora, 2016) e do criador (Neff, Wissinger \& Zukin, 2005), influenciando-se mutuamente. A competição por consumo também ocorre no campo simbólico e não no campo da utilidade prática (Lawrence \& Phillips, 2002; Khaire, 2017).

Com efeito, existe uma mistura ou indefinição entre produção e consumo neste empreendedorismo (Wilson \& Stokes, 2002). Criação e consumo se tornam altamente simbólicos (Davel \& Cora, 2016). Essa mistura contribui com a sensação de criatividade (Neff, Wissinger \& Zukin, 2005) e relaciona-se à interpretação coletiva, percepção estética-sensorial e inclusão em padrões de expressão, identidade e práticas culturais (Davel \& Cora, 2016), já que o consumo molda relações interpessoais (Zelizer, 2005). Assim, há a interpretação de valores simbólicos pelas experiências vividas, influenciando o processo criativo de construção de novos bens culturais e artísticos (Davel \& Cora, 2016).

O gosto de consumidores se relaciona ao lugar onde se socializaram e às coisas a que foram expostos (Khaire, 2017). Assim, os padrões de consumo associam-se à status social, grupos por padrões de gosto, em roupas, formas de entretenimento, em suma, identidades que associam grupos com senso individual de estilo (Boudieu, 2007). Estilo é termo chave na associação do conjunto de ideias a um produto, o que envolve a produção e a significação de textos que tornam o produto significativo, já que o estilo é construído coletivamente (Lawrence \& Philips, 2002). Estas decisões tomadas socialmente geram inovação, beleza e emoção (Lawrence \& Philips, 2002). Certas características de consumo por grupo, perfis e preferências (Khaire, 2017) dispõem de diferentes papeis na criação artística, como os papeis de consumidores especiais (por exemplo, os fãs) (Morris, 2014) e da crítica (Debenedetti, \& Larceneux, 2011; Khaire, 2017; Svejenova, Pedersen \& Vives, 2011). Destacar a singularidade do empreendedorismo artístico e cultural significa destacar a obra enquanto experiência de consumo subjetivamente transformativa (Davel \& Cora, 2016).

Inovação subjetiva da obra empreendedora. O valor cultural requer uma reflexão sobre a identificação de avanços genuínos na cultura, como avanços estéticos, sua mensuração e benefícios societais mais amplos (Cunningham \& Potts, 2015). A importância da inovação para o empreendedorismo é enfatizada desde a análise schumpteriana, pois é imprescindível para estudar aspectos de criação e mudança (Elias et al., 2018; Scherdin \& Zander, 2011; Swedberg, 2006). Na arte, a criatividade e a inovação são singulares (Scherdin \& Zander, 2011) porque o que elas trazem de inesperado, transformador e revolucionário é combustível para a evocação de sentimentos éticos e estéticos, que se traduzem em sonhos, descobertas e em imaginação (Frankelius, 2011). Consequentemente, a inovação artística é tão comum e economicamente relevante quanto a inovação de setores tradicionais (Frankelius, 2011). Porém, na medida em que as inovações artísticas diferem das inovações tecnológicas funcionais elas despontam como desafios e oportunidades únicos para o empreendedorismo (Islam, Endrissat \& Noppeney, 2016). Empreender passa a significar atuar na transformação, demandando robusto e fértil processo de produção (Meisiek \& Haefliger, 2011), criação (Scherdin \& Zander, 2011) e diversidade (Frankelius, 2011). 
No campo da cultura e das artes, as inovações são expressivas e comunicam estruturas culturais. Produtos criativos envolvem possibilidades estéticas, conceituais e emocionais abertas através do envolvimento com novas criações (Islam, Endrissat \& Noppeney, 2016). Portanto, o valor que artistas criam depende do grau de mudança de visões dominantes, trazendo emancipações, valor por significado. Isso situa a inovação como uma atividade centralmente humana que se apoia em contextos culturais para buscar o inusitado (Meisiek \& Haefliger, 2011). Em contextos culturais, a inovação é construída a partir da combinação entre aceitação do público (gosto) e avaliação da crítica (contribuições substanciais para o campo artístico). Essa combinação viabiliza a formação diferencial da arte na emergência de processos inovadores de renovação (Meisiek \& Haefliger, 2011; Menger, 2014; Becker, 1982; Bourdieu, 1993; 1999; 2007; Beuys, 2004). Neste sentido, a inovação coloca-se como intersubjetiva, ou seja, se faz por elementos compartilhados (Merleau-Ponty, 1999).

Esta inovação pode ser compreendida em termos de dissidência, reforço, aceitação cultural e compartilhamento de experiências (Becker, 1982), inovação aberta e formas sociais (Beuys, 2004), noção de campo social, competitividade e hierarquia (Bourdieu,1993). Com efeito, pesquisas recentes estudam a inovação (Islam, Endrissat \& Noppeney, 2016; Montagnino, 2020; Pedroni \& Volante, 2014) com base em aspectos de recombinação e reaproveitamento de materiais para a criatividade, reconfiguração de espaços, criação de invenções, cognição incorporada, simultaneidade entre novidade e familiaridade de inovações culturais (Islam, Endrissat \& Noppeney, 2016; Montagnino, 2020), disrupção, aceitação e transformação social (Montagnino, 2020), colaboração, esforço coletivo, conexões, criatividade coletiva e design participativo (Mitra, 2020; Montagnino, 2020). Ainda que devam ser considerados os processos de imitação do mercado, encontramos várias formas de exploração da inovação por meio da combinação de diversas técnicas: o ritmo cíclico de inovação (em tendências de renovação), seus efeitos positivos e negativos (as relações entre os aspectos de talento e comportamento de artistas empreendedores), processos de teste e erro e reiteração de sucesso (Menger, 2014).

Os conceitos de inovação cultural (Holt \& Cameron, 2010; Ravasi, Rindova \& Dalpiaz, 2012; Islam, Endrissat \& Noppeney, 2016) e de inovação estética (Dalpiaz, Rindova \& Ravasi, 2016; Lena \& Pachucki, 2013; Menger, 2014) emergem nas pesquisas. Como artistas são intérpretes do presente em tendências e gostos (Ártico \& Tamma, 2018), há tanto troca e mistura de valores e criação de inovação no pensamento e prática social (inovação cultural) quanto na arte em si (inovação estética). As inovações culturais e estéticas podem ser entendidas como as chamadas inovações leves (softs), e muitas vezes discretas, aquelas subjacentes aos setores culturais e que colocam constantes melhorias nos serviços, processos, responsabilidades e funcionalidades tanto quanto desenhos experimentais que afetam potencialmente todos os membros e outros setores da sociedade (Cunningham \& Potts, 2015). Valores subjetivos, pessoais e coletivos imbricam-se no mundo dos negócios para influenciar a vida artística e cultural (Formica, 2020). Este processo de imbricação gera mudanças na vida e imagem artística clássica (Menger, 2014), nas transformações no mundo do trabalho (Becker 1982; Caves, 2000; Eikhof \& Haunschild, 2006; Neff, Wissinger \& Zukin, 2005), produtos e serviços tradicionais (Àrtico \& Tamma, 2018), já que esta cultura se expandiu para atividades não artísticas (Eikhof \& Haunschild, 2006).

Essas mudanças transbordam para outros setores (spillover) (Cunningham \& Potts, 2015), envolvendo a inovação e gerando desenvolvimento, o que coloca o empreendedorismo como indispensável (Petrova, 2018). Como fatores de inovação econômica, social e cultural, identificamos mudanças pela mobilidade, colaboração e fertilização cruzada conferida pela classe criativa (Florida, 2012), também relacionada ao sentido cultural de lugar, à processos de 'culturalização' e estetização em áreas urbanas (Petrova, 2018). Trata-se da inovação estética e cultural na coletividade do sistema artístico (Menger, 2014) e social. Assim, a inovação cultural e estética aprofundam especificações da inovação movida pelo empreendedorismo no contexto da economia criativa como formas de inovação contemporâneas.

Lógica artística do processo empreendedor. As lógicas institucionais que governam o empreendedorismo na economia criativa e nos demais mercados são bastante distintas (Khaire, 2015). A 
lógica artística envolve a criação de autenticidade (Banks, 2006), oportunidades prioritariamente artísticas (Svejenova, Slavich \& Sondos, 2015) e se assenta nas identidades e nas características do trabalho artístico (Becker, 1982; Beuys, 2004; Bourdieu, 1996; Menger, 2014; Swedberg, 2006). A lógica artística flui com a inovação (Formica, 2020) e a gestão da incerteza, já que o trabalho artístico ressoa de forma individual (fluxo de gratificação individual) e coletiva (evidência social de prestígio acoplada a não utilitarismo) (Menger, 2014). Isso permite a constante renovação da produção e a perpetuação regenerada de poder de significação e sedução (Menger, 2014).

Um estilo de vida artístico demarca o perfil do chamado empreendedorismo boêmio-artístico em um processo de imbricação entre vida e trabalho vinculado ao fazer sem fronteiras claras (Eikhof \& Haunschild, 2006; Scherdin \& Zander, 2011). Neste contexto, o trabalho artístico, apesar de ainda muito devoto, subordinado e imbricado à vida pessoal, busca mais a autorrealização do que o sustento (Eikhof \& Haunschild, 2006). Esta visão, povoada pela deliberada subversão, contradição, afastamento e rejeição da ética, valores e normas dos princípios econômicos tradicionais e rígidos, valoriza distinções pessoais e certas tensões relacionadas a um estilo que expõe 'desvantagens' consideradas intoleráveis quando comparadas ao empreendedorismo tradicional (Eikhof \& Haunschild, 2006). Perfis e ações de egocentrismo, individualismo, narcisismo, ilusão, espontaneidade, mobilidade, necessidade de reconhecimento, respeito, melhoria contínua, aproveitamento da vida e rejeição dos princípios econômicos (Eikhof \& Haunschild, 2006) repercutem na prática profissional como na desconfiança em relação à artistas na concessão de financiamentos bancários (Borin, Donato, \& Sinapi, 2018; Wilson, \& Strokes, 2002), por exemplo. Por outro lado, estes perfis são tensionados com o sentimento de pertença social, necessidade de coletivismo, rede (mesmo como fonte de oportunidade) e a necessidade de comercializar ativamente arte como inclusão na produção da arte pela arte (Eikhof \& Haunschild, 2006), criar inovação lidando com a incerteza, as ilusões (Menger, 2014) e as possessões artísticas (Scherdin \& Zander, 2011). A necessidade de comercialização destes perfis justifica a necessidade de autopromoção por meio de práticas boêmias, pela compreensão, estilização e venda de singularidades pessoais (por exemplo, roupas extravagantes, gestos ou hábitos típicos) (Eikhof \& Haunschild, 2006). Ou seja, o artista vai acoplando o marketing à sua personalidade de artista (Rentschler, 2007).

Caves (2000) e Florida (2012) deram indícios da relação entre economia e identidades artísticas (Bourdieu, 1996) nas indústrias criativas que dependem da motivação artística como seu principal recurso produtivo (Eikhof \& Haunschild, 2006). Antes disso, a identidade artística já era concebida de forma plural: desejo pela arte e trabalho como extensão natural, perfil visionário, transformador, subversivo, contendo alguns 'mavericks' culturais, certo grau de marginalidade, capilaridade e senso de aproveitamento (Becker 1982), formas de capital (Boudieu, 1996), aspectos de criatividade e inovação (Beuys, 2004). Na tensão das visões, por exemplo, para Marx (1844) o trabalho artístico era modelo de não alienação, uma atividade concreta pela qual tem-se a realização da liberdade pela externalização e objetificação de poderes (Menger, 2014). Nesse sentido, a lógica do trabalho artístico, seria em essência crítico e de realização de liberdade (Menger, 2014). No entanto, no contexto atual da economia criativa, a maneira como identidades artísticas são mobilizadas como forma de trabalho empreendedor, transmitindo imagens de diversão e prazer, encobertaria dificuldades e riscos (Neff, Wissinger \& Zukin, 2005; Ellmeier, 2003) e abriria novos debates. Estes debates incluem a crítica à visão romântica e renascentista sobre o artista, formas de desigualdades em torno do talento (Menger, 2014), as discussões em torno de noções de estrelado (Currid-Halkett, 2015) e a relação com a ideia de genialidade (Koivunen \& Rehn, 2009) ou criatividade individual, ainda presente, porém já contestada por visões sociais (Cattani; Ferriani \& Colucci, 2015).

A lógica artística revelaria ao menos duas interpretações: (a) individualismo hedonista (como tipo social de criação infinita e renúncia da segurança material) e (b) heroísmo social comprometido com a arte superior que leva a um interesse geral de coletividade pelo prazer e bem-estar das gerações futuras (Menger, 2014). São as contradições entre indivíduo e coletivo. Mesmo que os valores de coletividade, vínculo, retorno social, sentimento de pertença e necessidade de rede (Eikhof \& Haunschild, 2006) estejam presentes, os artistas também operam em regimes de produção individualistas pelo seu interesse 
de obter pleno emprego, sucesso e autorrealização (Eikhof \& Haunschild, 2006; Menger, 2014). Consequentemente, a arte envolve redes de luta na busca de posições desejadas (Bourdieu, 1993) a partir de relações colaborativas e antagônicas, posto que há processos competitivos (Menger, 2014) de diferenciação e renovação estéticos (Meisiek, \& Haefliger, 2011). Há acordos entre grupos dominantes e excludentes sobre as fronteiras do que é arte, forçando atores subversivos a buscarem adaptações (Pedroni \& Volante, 2014). Assim, tanto o individualismo pode ser visto como o princípio e resultado de competição entre artistas na sua sistemática busca por originalidade estética, quanto como o produto da ideologia expressionista e romântica através da qual o artista é o individual por excelência (Menger, 2014).

Lógica cultural do processo empreendedor. Enquanto a lógica artística nos leva, em síntese, para o campo individual, competitivo e da inovação, a lógica cultural nos leva para o campo coletivo, cooperativo e da preservação. Toda a arte é cultura, mas nem toda cultura é arte. Assim, o valor cultural torna-se mais abrangente do que o valor artístico (Birnkrau, 2018). A lógica cultural expande a visão existente na lógica artística: enquanto a arte coloca-se como criação e expressão, a cultura coloca-se como criação, expressão e troca direta para além da arte (Marriot, \& Miller, 2012). A cultura refere-se às esferas sociais, funcionais, comunitárias, familiares, afetivas. Inclui também altruísmo social, interesses coletivos (Campos \& Davel, 2018; Cora, 2016; Ettinger, 2015; Judice \& Furtado, 2014; Reis \& Zille; 2019) e outras formas de entender a arte como bem público que precisa ser compartilhado (Toghraee \& Monjezi, 2017). Trata-se de um modo ético de práticas comunais e coletivas (Banks, 2006).

$\mathrm{Na}$ comparação entre definições de empreendedorismo na cultura e nas artes, observamos uma variabilidade de interesses e motivações. O conceito de empreendedorismo sociocultural tenta aliar a visão sociocultural à visão cultural-artística para esclarecer a necessidade de enfrentamento de desafios sociais através do uso da arte (Birnkrau, 2018; Scherdin \& Zander, 2011; Svejenova, Slavich \& Sondos, 2015). Observamos que existem aproximações do empreendedorismo cultural e artístico ao empreendedorismo social (Birnkrau, 2018; Campos \& Davel, 2017), empreendedorismo artesanal (Hoyte, 2019) e empreendedorismo comunitário, étnico e territorial. A cultura refere-se também a vínculos comunitários, territoriais, históricos e contextuais (Lounsbury \& Glynn, 2019; Khaire, 2015; 2017). A relação entre empreendedorismo e território destaca o conceito de cidades criativas (Florida, 2012; Klamer, 2011; Toghraee \& Monjezi, 2017). Além disso, há a relação temporal, espacial, local entre a penetração da cultura e seu sentido na geração de novas ideias que moldam o empreendedorismo (Khaire, 2017; Lounsbury, \& Glynn, 2019).

Instituições culturais tradicionais (como aquelas sem fins lucrativos) apoiam-se no empreendedorismo para gerir crises e contribuir com o desenvolvimento de áreas periféricas (Papathanasiou-Zuhrt, Russo \& Kutsikos, 2019). Fala-se em empreendedorismo patrimonial (heripreneurship), com discussões sobre financiamento público, a peculiaridade de bens que não são mercadorias tradicionais, obras individuais com narrativas de valor simbólico e educacional (Papathanasiou-Zuhrt, Russo \& Kutsikos, 2019). A lógica cultural envolve uma percepção mais refinada do empreendedorismo quanto à aspectos organizacionais e de gestão que vão desde a natureza e finalidade das organizações à aspectos de produção, distribuição e consumo.

Lógica paradoxal do processo empreendedor. Paradoxos ou tensões que envolvem o empreendedorismo nas artes e na cultura (Banks et al., 2000; Eikhof \& Haunschild, 2006; 2007; Ellmeier, 2003; Formica, 2020; Khaire, 2017; Scherdin \& Zander, 2011; Swedberg, 2006; Toghraee \& Monjezi, 2017) são frequentemente apontados pelas pesquisas, apesar de pouco desenvolvidos. O paradoxo primordial e singular tratado no campo das indústrias criativas é a tensão arte e comércio (Caves, 2000; Khaire, 2015; Lawrence \& Philips, 2002; Khaire, 2017). Esta tensão posiciona a ideia de arte pela arte em negação às visões econômicas, uma tensão amplamente pesquisada por sociólogos e outros estudiosos do mundo das artes (Bourdieu, 2007; 1996; Ellmeier, 2003).

A arte é uma esfera de desempenho estético que se contrapõe à orientação econômica e utilitarista do mercado (Elias et al., 2018; Ellmeier, 2003; Eikhof \& Haunschild, 2006; 2007; Scherdin \& Zander, 2011), 
prosperando não apenas em ser arte, mas também por não ser exclusivamente econômica (Bourdieu, 1993; 2007; Eikhof \& Haunschild, 2006), não se resumindo à questões de oferta, demanda e distribuição de produtos e insumos (Klamer, 2011). Em Marx (1844) as relações sociais capitalistas intrometem-se nas artes a reduzindo à mercadoria, trabalho artístico à trabalho assalariado (Menger, 2014). Esta redução imprime a perda de valor simbólico e o ganho de valor econômico na arte (Menger, 2014). Como pertinente ao mundo econômico de exploração de novas oportunidades e geração de riquezas, o empreendedorismo renova esta discussão reinserindo ou intensificando valores econômicos no mundo cultural (Ellmeier, 2003). Por outro lado, visões colocam as tensões como provocadas pelos valores culturais (Toghraee \& Monjezi, 2017), que são preservacionistas e críticos, enquanto que, na prática, mesmo que consumidores realizem a compra motivados por prazer estético, eles ainda precisam pagar um valor monetário (Kolb, 2015).

Com isso, o empreendedorismo cultural depende de um jogo constante de articulação e equilíbrio entre diferentes sistemas de valores (Petrova, 2018), necessitando identificar e compreender os valores culturais de tal maneira que os parâmetros financeiros atuem como meios (Toghraee \& Monjezi, 2017). As pesquisas buscam superar esses paradoxos, integrando estratégias como a de produtos baseados em cultura (Ártico \& Tamma, 2018), estilo de vida (Eikhof \& Haunschild, 2006; 2007) ou design análogo às narrativas persuasivas (Khaire, 2015). Este conflito e suas consequências podem ser estudados de maneira mais persuasiva entre os artistas que precisam comercializar e gerenciar sua própria força de trabalho (Eikhof \& Haunschild, 2006; Ellmeier, 2003).

As tensões evidenciam as sutis diferenças de perspectivas que variam entre a maior valorização cultural e riqueza de significados (Banks et al., 2000; Davel \& Cora, 2016) à geração de riquezas monetárias (Wilson \& Strokes, 2002). Consequentemente, outras tensões emergem, já que variam entre linguagens artísticas e culturais como na moda, que dispõe mais de dificuldades estéticas que o equilíbrio entre arte e comércio (Lawrence \& Philips, 2002). Fala-se em tensão entre exclusão versus inclusão, entre cotidiano versus ocupação (Eikhof \& Haunschild, 2006; 2007), criatividade versus controle (Eikhof \& Haunschild, 2006; 2007), indivíduo versus contexto (Lange, 2018), diversão versus risco (Neff, Wissinger \& Zukin, 2005), linguagem empresarial versus linguagem artística, diferentes tipos de parâmetros de confiabilidade entres os campos versus estratégia, rivalidade versus colaboração, trabalho versus ócio, individualismo versus trabalho colaborativo (Wilson \& Strokes, 2002; Lange, 2018), criatividade versus crescimento (Toghraee \& Monjezi, 2017), competividade versus coletivismo, arte lucro versus arte liberdade (Menger, 2014).

Lógica arriscada inerente ao processo empreendedor. A tolerância em assumir riscos é percebida como uma orientação individual durante a tomada de decisão em situações de criação perante à exposição ao consumo (Lingo \& Teeper, 2013; Poorsoltan, 2012). Riscos e incertezas são subjacentes ao trabalho empreendedor e artístico (Menger, 2014; Scherdin \& Zander, 2011; Toghraee \& Monjezi, 2017), por serem alvos constantes de mudanças culturais de gosto e por envolverem significados complexos (Lawrenece \& Philips, 2002).

São características dessa situação exacerbada de risco no campo do empreendedorismo artístico e cultural: (a) as criações expressas como experiências que não podem ser testadas (Davel \& Cora, 2016), (b) o sucesso do produto ou da experiência como imprevisível, em relação à inovação (Neff, Wissinger \& Zukin, 2005) e imaginação, já que a obra é imaginada e assim perseguida sem certeza de sucesso (Formica, 2020), (c) a competição no trabalho artístico (Menger, 2014), (d) a assimetria da informação (ou o não fornecimento de segurança adequada para investidores), (e) a não confiabilidade gerada pela imagem de magia/mistério, de juventude de setores e sua compreensão a respeito das capacidades de gestão, ruídos no diálogo pela linguagem diferenciada entre arte e mercado (Wilson \& Stokes, 2002). A incerteza envolve duas dimensões indissociáveis: a intrínseca (que caracteriza a lacuna entre esforços e o objetivo atingido, o que traz prazer e ansiedade) e a estratégica e social (o valor da obra artística no reconhecimento de talentos é indissociável de valorização comparativa e consequentemente de testes competitivos) (Menger, 
2014). Não há como ter certeza de sucesso, por exemplo, pelo aspecto comparativo do talento (Menger, 2014).

A gestão de incerteza nas estratégias de empreendedorismo artístico e cultural envolve alguns processos (Menger, 2014; Petrova, 2018; Poorsoltan, 2012): (a) a gestão de fatores pessoais, como a tolerância à riscos (conforto e gosto em situações complexas), o lócus interno de controle e a adaptabilidade em diferentes campos; (b) a gestão de fatores claramente estéticos, como estilos (linguagens, capitais e pessoas) e tempo de amadurecimento estético; (c) a gestão de sistemas de valorização colaborativos e informais, como certificações, status, portifólio, reputação e laços; (d) a gestão de comportamento de mercado como a força do sistema de estrelado, inovação, imitação, legislação (contratos flexíveis), controle de custos (subcontratação, aluguel etc.) e controle informacional; (e) gestão da profissionalização, como a educação.

Na relação entre consumo e incerteza, retornamos à questão do valor, fruto de um complexo mecanismo de seleção por avaliações comparativas e hierárquicas que expressam como grupos consolidam um sistema de valorização estética. Isso se revela posteriormente com a sensibilidade da demanda. Demanda insensível gera menores riscos porque ela não é volátil à qualidade, o que impede a substituição do artista e evita competição desequilibrada. Em contrapartida, demanda sensível amplia riscos e incertezas (Menger, 2014).

\subsection{Temáticas nas pesquisas sobre empreendedorismo na arte e na cultura}

Algumas temáticas foram identificadas como centrais na produção acadêmica sobre empreendedorismo, cultura e arte (Tabela 1). Outras temáticas são emergentes e aparecem mais pulverizadas na produção acadêmica: perspectiva territorial (comunitária, rural, regional, urbana) e rede (Hausmann \& Heinze, 2016; Qian \& Liu, 2018; Toghraee \& Monjezi, 2017), importância e criação do contexto (Petrova, 2018), imaginação empreendedora e ignorância criativa (Formica, 2020), sucesso e fracasso (Hausmann \& Heinze, 2016; Konrad, 2013; Klamer; 2011; Menger, 2014; Kuznetsova-Bogdanovits, RanczakowskaLjutjuk \& Kiitsak-Prikk, 2018; Riot, 2013), brincadeira e ludicidade (Formica, 2020), institucionalização da conversa e storytelling (Borghoff, 2018; Lounsbury \& Glynn, 2019; Klamer, 2011; Khaire, 2015; Lindkvist \& Hjorth, 2015; Eikhof \& Haunschild, 2006; Formica, 2020), objetos (Khaire, 2015) e tendências (Hausmann \& Heinze, 2016; Preece, 2011; Toghraee \& Monjezi, 2017).

\begin{tabular}{|l|l|}
\hline Temáticas & Subtemas e Principais Referências \\
\hline Perfil & $\begin{array}{l}\text { Identidade empreendedora, motivações e finalidades. Teorias dos traços. Perfil persuasivo, } \\
\text { inovador, gerador de mudanças, tolerante à riscos, multifacetado. Identificação de tipos de } \\
\text { agentes como empreendedores. O perfil é fator de sucesso (Chang \& Wyszomirski, 2015; Elias } \\
\text { et al., 2018; Eikhof \& Haunschild, 2006; 2007; Hausmann \& Heinze, 2016; Ellmeier, 2003; } \\
\text { Khaire, 2015; 2017; Klamer, 2011; Konrad, 2018; Leadbeater \& Oakley, 1999; Lingo \& Teeper, } \\
\text { 2013; Menger, 2014; Neff, Wissinger \& Zukin, 2005; Petrova, 2018; Preece, 2011; Poorsoltan, } \\
\text { 2012; Rae, 2005; Riot, 2013; Scott, 2012; Toghraee \& Monjezi, 2017; Toscher, 2020; Werthes, } \\
\text { Mauer \& Brettel, 2017; Wilson \& Stokes, 2002; 2004, 2005). }\end{array}$ \\
\hline Organização e & $\begin{array}{l}\text { Intraempreendedorismo em organizações, criação de novas organizações. Instituições culturais, } \\
\text { gestão de espaços, trabalhos individuais, projetos e atividades temporárias. Atividades de }\end{array}$ \\
& $\begin{array}{l}\text { intermediação e incubação. Habilidades gerenciais, profissionais e de rede. Conhecimento de } \\
\text { mercado, marketing, finanças, planejamento, startups (Araujo \& Davel, 2019; Borin, Donato \& } \\
\text { Sinapi, 2018; Ellmeier, 2003; Essig, 2017; Hagoort, 2007; Khaire, 2015; 2017; Konrad, 2018; } \\
\text { Hausmann \& Heinze, 2016; Kolsteeg, 2013; Leadbeater \& Oakley, 1999; Lingo \& Teeper, 2013; } \\
\text { Loy \& AAgeson; 2018; Marins \& Davel, 2019; Menger, 2014; Papathanasiou-Zuhrt, Russo \& } \\
\text { Kutsikos, 2018; Preece, 2015; 2011; Reis \& Zille, 2020; Ruiz, Colbart \& Hinna, 2017; Scherdin } \\
\text { \& Zander, 2011; Toghraee \& Monjezi, 2017; Wilson \& Stokes, 2002). }\end{array}$ \\
\hline Educação & $\begin{array}{l}\text { Estudos e oferta acadêmica limitados. Análises comparativas entre países. Crítica à ausência do } \\
\text { empreendedorismo artístico-cultural em currículos. Crítica a currículos tradicionais. Críticas na } \\
\text { tensão arte versus comércio. Conteúdos sobre identidade, tecnologia social e desenvolvimento } \\
\text { dos territórios (Araujo \& Davel, 2019; Campos \& Davel, 2017; Birnkrau, 2018; Brandenburg, } \\
\text { Roosen \& Veenstra, 2016; Bryan \& Harris, 2015; Ellmeier, 2003; Essig, 2017; Hausmann \& }\end{array}$ \\
\hline
\end{tabular}




\begin{tabular}{|c|c|}
\hline & $\begin{array}{l}\text { Heinze, 2016; Klamer, 2011; Konrad, 2018; Korzen, 2015; Kuznetsova-Bogdanovits, } \\
\text { Ranczakowska-Ljutjuk \& Kiitsak-Prikk, 2018; Menger, 2014; Stefanovic, 2018; Thom, 2014; } \\
\text { 2016; 2017; Toghraee \& Monjezi, 2017; Toscher, 2020; White, 2015; Wilson \& Strokes, 2002). }\end{array}$ \\
\hline Políticas & $\begin{array}{l}\text { Políticas públicas, privadas e sociais. Carência de aprofundamento científico. Particularidades } \\
\text { como riscos e dificuldades de autossutentabilidade. Estímulo à pesquisa e ensino. Infraestrutura } \\
\text { e tecnologia. Avaliação de impactos, regulação e prioridades. Análise de gastos e financiamentos } \\
\text { (Ártico \& Tamma, 2018; Banks, et al., 2000; Borin, Donato \& Sinapi, 2018; Campos \& Davel, } \\
\text { 2017; Preece, 2011; Haussman \& Heize, 2006; DiMaggio, 1982; Ellmeier, 2003; Essig, 2017; } \\
\text { Konrad, 2018; Kuznetsova-Bogdanovits, Ranczakowska-Ljutjuk \& Kiitsak-Prikk 2018; } \\
\text { Marriott \& Miller, 2012; Patten, 2016; Qian \& Liu, 2018; Reis \& Zille, 2020; Ruiz, Colbart \& } \\
\text { Hinna, 2017; Stefanovic, 2018; Toghraee \&, Monjezi, 2017; Wilson \& Stokes, 2002; Yoon, } \\
\text { 2017). }\end{array}$ \\
\hline $\begin{array}{l}\text { Singularidades } \\
\text { das culturas e } \\
\text { linguagens }\end{array}$ & $\begin{array}{l}\text { Crítica à carência de enfoque na fragmentação e heterogeneidade. Empreendedorismo como } \\
\text { forma de singularização. Informações socioculturais, setoriais, econômicas e de consumo. } \\
\text { Variação de paradoxos arte-comércio entre linguagens artísticas (Banks, et al., 2000; Campos \& } \\
\text { Davel, 2018; Cunningham \& Potts, 2015; Guerra \& Paiva Junior, 2011; Eikhof \& Haunschild, } \\
\text { 2006; Gillespie, 2007; Pechlaner, \& Innerhofer, 2019; Hausmann \& Heinze, 2016; Khaire, 2015; } \\
\text { Klamer, 2011; Konrad, 2018; Kuznetsova-Bogdanovits, Ranczakowska-Ljutjuk \& Kiitsak- } \\
\text { Prikk, 2018; Menger, 2014; Moris, 2014; Marins \& Davel, 2019; Neff, Wissinger \& Zukin, 2005; } \\
\text { Oliveira et al., 2016; Svejenova, Slavich \& Sondo, 2015; Wilson \& Strokes, 2002; 2004). }\end{array}$ \\
\hline Tecr & $\begin{array}{l}\text { Tecnologias digitais contemporâneas como fonte de criatividade, estética e desafios como } \\
\text { inteligência artificial e realidade aumentada. Políticas infraestruturais frente à alteração da } \\
\text { produção, distribuição, direitos autorais, mudança no papel de consumo e autonomia. Crítica à } \\
\text { ausência da natureza humana da tecnologia (Formica, 2020; Lingo \& Teeper, 2013; Morris, } \\
\text { 2014; Qian \& Liu, 2018; Toghraee \& Monjezi, 2017; Wilson \& Strokes, 2002; Yoon, 2017). }\end{array}$ \\
\hline $\begin{array}{l}\text { Trabalho e } \\
\text { carreira }\end{array}$ & $\begin{array}{l}\text { Crítica a perfis e formas de trabalho nos diferentes setores (carreira, estratégia, portfólio, risco). } \\
\text { Relações com o contexto local e identidade. Trabalho bom versus trabalho ruim (Ellmeier, 2003; } \\
\text { Guo \& Wyszomirski, 2018; Leadbeater \& Oakley, 1999; Lingo \& Teeper, 2013; Morris, 2014; } \\
\text { Neff, Wissinger \& Zukin, 2005). }\end{array}$ \\
\hline
\end{tabular}

Tabela 1: Principais temáticas na produção acadêmica sobre empreendedorismo na cultura e nas artes Fonte: Autores

\subsection{Impactos do empreendedorismo na arte e na cultura}

Não identificamos estudos aprofundados ou sistematizados sobre impactos, apesar de serem recorrentemente citados de forma indireta ou pontual. A partir de uma análise mais densa e atenta da produção acadêmica, foi possível sistematizar e categorizar um conjunto de impactos (Tabela 2). O empreendedorismo nas artes e na cultura apresenta grande potencial (Leadbeater \& Oakley 1999), pois enfrenta uma velocidade acirrada e intensa de mudanças (Cunignham, \& Potts, 2015, Ellmeier, 2003; Klamer, 2011; Khaire, 2017). Os impactos são intencionais, não intencionais, planejados e não planejados, diretos e indiretos, negativos e positivos, tangíveis e intangíveis (Toghraee \& Monjezi, 2017; Petrova, 2018). Envolvem pessoas, organizações e regiões desde empreendedores atuais à potenciais, gestores culturais, artistas, críticos, educadores, líderes comunitários, formadores de políticas e acadêmicos. Não encontramos estudos significativos para a categoria ambiental.

\begin{tabular}{|l|l|}
\hline Tipo & \multicolumn{1}{|c|}{ Impactos } \\
\hline Econômico & $\begin{array}{l}\text { Positivos diretos: Criação de micro, pequenas e médias empresas. Aumento e diferenciação de } \\
\text { empregos como aqueles intermediários. Produção criativa. Investimentos públicos e privados. } \\
\text { Mitigação de falhas de mercado. Lucro e riqueza individual. Novos mercados (Ártico \& Tamma, } \\
\text { 2018; Khaire, 2017; Leadbeater \& Oakley, 1999; Marriott \& Miller, 2012; Neff, Wissinger \& } \\
\text { Zukin, 2005; Petrova, 2018; Toghraee \& Monjezi, 2017; Wilson \& Stokes, 2002). } \\
\text { Positivos indiretos: Aumento do PIB. Desenvolvimento e crescimento endógeno em pequenas } \\
\text { e rurais comunidades de países em desenvolvimento. Mudanças regionais urbanas. Incremento } \\
\text { de setores como o do turismo. Economia global (Ártico \& Tamma, 2018; Campos \& Davel, } \\
\text { 2018; Cunignham \& Potts, 2015; Ellmeier, 2003; Formica, 2020; Jones, Lorenzen \& Sapsed, } \\
\text { 2015; Khaire, 2015; Klamer, 2011; Leadbeater \& Oakley, 1999; Loy \& AAgeson, 2018; Marriott } \\
\text { \& Miller, 2012; Petrova, 2018; Qian \& Liu, 2018); Stefanovic, 2018; Toghraee \& Monjezi, 2017). } \\
\text { Negativos: Precarização do trabalho e exploração pelo mercado: baixa remuneração, alta carga } \\
\text { de trabalho, riscos. Aumento de custos. Competição. Imposição de ideologias econômicas não }\end{array}$ \\
\hline
\end{tabular}




\begin{tabular}{|c|c|}
\hline & $\begin{array}{l}\text { reconhecidas (Banks, et al., 2000; Ellmeier, 2003; McRobbie, 2011; Menger, 2014; Neff, } \\
\text { Wissinger \& Zukin, 2005; Kuznetsova-Bogdanovits, Ranczakowska-Ljutjuk \& Kiitsak-Prikk, } \\
\text { 2018; Patten, 2016; Ruiz, Colbart \& Hinna, 2017). }\end{array}$ \\
\hline Cultural & $\begin{array}{l}\text { Positivos diretos: Realização e sustentabilidade de valores culturais e sociais. } \\
\text { Estímulo à manutenção de tradições como parte da identidade. Fortalecimento de laços sociais. } \\
\text { Difusão da cultura popular. Formação de organizações populares sem fins lucrativos. } \\
\text { Experiências cognitivas, emocionais e multissensoriais de longa duração para diferentes públicos } \\
\text { (Marriott \& Miller, 2012; Khaire, 2017; Klamer, 2003; 2011; Judice \& Furtado, 2014). } \\
\text { Positivos indiretos: Alteração de paisagens culturais. Agregação de valor cultural em produtos } \\
\text { tradicionais. Criação de empresas com valor social e cultural. Geração de mudança, valorização e } \\
\text { transformação. Inovação cultural e estética (Araujo \& Davel, 2019; Ártico \& Tamma, 2018; } \\
\text { Cunignham \& Potts, 2015; Jones, Lorenzen \& Sapsed, 2015; Formica, 2020; Klamer, 2011; } \\
\text { Marins \& Davel, 2019; Petrova, 2018; Swedberg, 2006). } \\
\text { Negativos: Desproteção, mercantilização, empreendedorização e economização da cultura e do } \\
\text { patrimônio cultural. Perpetuação de valores excludentes e opressores. Possível entorpecimento e } \\
\text { perda de autenticidade em produtos, serviços e organizações. Influência negativa em atitudes e } \\
\text { comportamento dos consumidores. Valores como impositores de empecilhos ao comportamento } \\
\text { empreendedor (Ártico \& Tamma, 2018; Ellmeier, 2003; Toghraee \& Monjezi, 2017). }\end{array}$ \\
\hline Artístico & $\begin{array}{l}\text { Positivos diretos: Ampliação e diversificação de manifestações artísticas locais. } \\
\text { Criação de organizações. Oferta de artistas independentes. Alteração das redes locais. } \\
\text { Fortalecimento de identidades artísticas. Resposta emocional positiva: alegria, felicidade, } \\
\text { gentileza. Geração de interpretações, significados e habilidades criativas. Preservação de valores. } \\
\text { Autenticidade. Formação de senso crítico e engajamento social (Davel \& Cora, 2016; } \\
\text { Cunningham \& Potts, 2015; Formica, 2020; Lingo \& Teeper, 2013). } \\
\text { Positivos indiretos: Externalidades como capital humano criativo. Mudanças ecológicas, sociais, } \\
\text { tecnológicas, identitárias, ambientais, pessoais, comunitárias. Imbricação de perspectivas } \\
\text { consolidando a arte empreendedora (Cunningham \& Potts, 2015; Davel \& Cora, 2016; Formica, } \\
\text { 2020; Lingo \& Mahony, 2010). } \\
\text { Negativos: Restrita e supervalorizada seleção de artistas. Restrição de limites à criatividade, } \\
\text { autenticidade, senso crítico e engajamento social. Desrespeito à diversidade com imposição de } \\
\text { valores homogêneos. Competição e homogeneização de projetos. Redução da atmosfera criativa } \\
\text { frente à projetos de urbanização. Tensões entre artistas e estruturas de apoio com aumento de } \\
\text { disputas de poder. Fortalecimento de dicotomias: nicho ou massa, bem ou performance, sustento } \\
\text { ou riqueza, padrão e experiência íntima (Lingo, \& Teeper, 2013; Patten, 2016; Stefanovic, 2018). }\end{array}$ \\
\hline Social & $\begin{array}{l}\text { Positivos diretos: Projeção, retorno social e coesão social. Diversificação da oferta de } \\
\text { entretenimento local, questionamento e transformação de valores sociais. Ampliação da sensação } \\
\text { de prazer e felicidade (Ártico \& Tamma, 2018; Birnkrau, 2018; Ellmeier, 2003; Kuznetsova- } \\
\text { Bogdanovits, Ranczakowska-Ljutjuk \& Kiitsak-Prikk, 2018; Petrova, 2018; Leadbeater \& Oakley, } \\
\text { 1999; Marriott \& Miller, 2012; Shiray, Carvalho \& Afonso, 2017). } \\
\text { Positivos indiretos: Qualidade de vida e desenvolvimento comunitário. Cidadania. } \\
\text { Mudança qualitativa de espaços vulneráveis. Integração institucional (Cunningham \& Potts, 2015; } \\
\text { Marriott \& Miller, 2012; Petrova, 2018; Shiray, Carvalho \& Afonso, 2017; Yoon, 2017). } \\
\text { Negativos: Ampliação da exclusão de setores e indivíduos marginalizados: idosos, mulheres, } \\
\text { etnias. Precarização e exploração do trabalho. Concentração em áreas centrais, desatenção às } \\
\text { periferias. Falta de diversidade (Banks, et al., 2000; Ellmeier, 2003; Ettinger, 2015; McRobbie, } \\
\text { 2011; Neff, Wissinger \& Zukin, 2005; Lingo \& Teeper, 2013); Patten, 2016; Ruiz, Colbart \& } \\
\text { Hinna, 2017; Toghraee \& Monjezi, 2017). }\end{array}$ \\
\hline Político & $\begin{array}{l}\text { Positivos diretos: Geração e atualização de políticas de estímulo e regulação para o } \\
\text { empreendedorismo, arte e cultura, educação empreendedora e territórios (Klamer, 2011; } \\
\text { Ellmeier, 2003; Kuznetsova-Bogdanovits, Ranczakowska-Ljutjuk \& Kiitsak-Prikk, 2018; Patten, } \\
\text { 2016; Stefanovic, 2018). } \\
\text { Positivos indiretos: Mitigação de falhas do mercado. Estímulo à educação empreendedora } \\
\text { especializada. Estímulo à identidade local, regional, nacional (Jones, Lorenzen \& Sapsed, 2015; } \\
\text { Marriott \& Miller, 2012). } \\
\text { Negativos: Redução de responsabilidades financeiras do Estado. Aumento da pressão por } \\
\text { obtenção de lucro em instituições culturais. Homogeneidade e desatualização de políticas. } \\
\text { Desarticulação institucional. Seletividade e favoritismo de setores e projetos (Chang \& } \\
\text { Wyszomirski, 2015; Ellmeier, 2003; Klamer, 2011; Kolsteeg, 2013; Kuznetsova-Bogdanovits, } \\
\text { Ranczakowska-Ljutjuk \& Kiitsak-Prikk, 2018; Patten, 2016; Stefanovic, 2018). }\end{array}$ \\
\hline
\end{tabular}

Figura 2: Impactos do empreendedorismo na arte e na cultura

Fonte: Autores. 


\subsection{Forças e carências na produção acadêmica}

Algumas forças e carências foram identificadas na produção acadêmica. A primeira força refere-se à construção de um campo de pesquisas que ganha espaços estruturados, progressivamente, em revistas acadêmicas. A segunda força provém do processo de singularização de linguagens artísticas, que atentam para a importância da diversidade e diversificação dentro do empreendedorismo nas artes e na cultura. Isso requer transdisciplinaridade. A terceira força é a criatividade, elemento amplamente discutido, mas que atua como energia onipresente em termos, por exemplo, de singularidades e impactos.

As carências encontradas na produção acadêmica podem ser concebidas na forma de dois grandes desafios: teórico e metodológico. O desafio teórico envolve várias carências: (a) de visões críticas, (b) de transdisciplinaridade, (c) de um debate onto-epistemológico e (d) de enfoque na estética como uma perspectiva promissora.

A primeira carência é que poucos estudos são críticos, embora muitos exponham críticas. Alguns exemplos de críticas apontadas nas pesquisas são: valores neoliberais e função do Estado (Ellmeier, 2003; McRobbie, 2011), retórica positiva do desenvolvimento (Ellmeier, 2003; Essig, 2017; Neff, Wissinger, \& Zukin, 2005; Patten, 2016), conotações negativas sobre o empreendedorismo (Stefanovic, 2018; Toghraee \& Monjezi, 2017), lentes usadas na análise (Swedberg, 2006), marginalização de grupos sociais (Ellmeier, 2003; Ettinger, 2015; Neff, Wissinger \& Zukin, 2005), não preservação de valores (Lingo \& Teeper, 2013; Patten, 2016; Stefanovic, 2018), precarização do trabalho (Banks, et al. 2000; McRobbie, 2011; Ellmeier, 2003), imposição de valores homogêneos (Patten, 2016), concentração em áreas centrais (Lingo, \& Teeper, 2013; Patten, 2016), falta de inclusão sobre as formas de empreendedorismo no mundo (Ruiz, Colbart, \& Hinna, 2017; Toghraee, \& Monjezi, 2017); formas inadequadas de educação empreendedora (Birnkrau, 2018; Bryan, \& Harrys, 2015; Essig, 2015, 2017; Thom, 2015; 2017).

A segunda carência é de consolidação de uma perspectiva teórica que integre e combine várias disciplinas do conhecimento, como as ciências sociais, humanas, artísticas, culturais, administrativas, organizacionais, comunicacionais, históricas, econômicas, educacionais, políticas, urbanas. Essa integração e combinação poderá proporcionar um melhor entendimento do empreendedorismo artísticocultural, de suas singularidades, tensões e enriquecer as temáticas, que precisam ser mais singularizadas, para além de meras extrapolações do campo do empreendedorismo.

A terceira carência é relativa à consideração de questões onto-epistemológicas que permitam uma melhor coerência teórica para destacar as singularidades do campo artístico-cultural. Ontologias relacionais, coletivistas, processuais e sensíveis se adequam à análise do empreendedorismo cultural por sua especificidade relacional, subjetiva e simbólica (Klamer, 2011). Orientações ontoepistêmicas baseadas na perspectiva da prática (Gherardi, 2019; Reckwitz, 2007) também podem abrir novos caminhos de pesquisa.

A quarta carência é a da estética como perspectiva ontoepistêmica, teórica e metodológica promissora para renovar a pesquisa sobre o empreendedorismo cultural e artístico. Estudos reconhecem a necessidade e a importância de preocupações políticas, corpóreas e estéticas no estudo do empreendedorismo das artes e cultura, porém, ainda são escassos os estudos que se aprofundam na análise estética (Elias et al., 2018). A lógica estética molda o campo cultural (Laan \& Kuipers, 2016). A potência da estética em renovar pesquisas futuras se deve a vários fatores:

- A natureza intangível, invisível, líquida ou escondida (Cunningham \& Potts, 2015) entre dimensões econômicas e estéticas, incompreensíveis à mecanismos socioeconômicos que sustentam a criação (Menger, 2014).

- A complexidade de um conhecimento já desafiante dentro de organizações por tratar de subjetividades, sensações e julgamentos (Strati, 2007, 2019; Taylor \& Hansen, 2005; Warren, 2008). 
- A complexidade deste conhecimento em cadeia ou fronteiras fluidas (Wilson, \& Strokes, 2002), abertas e diversas de empreendedorismo nas artes e cultura (diferentes setores e suas intercessões, relacionamentos, redes), o que reduz a vantagem da maior clareza pela intensidade das trocas estéticas (Banks et al., 2000).

- A complexidade do equilíbrio na tensão entre valor estético e monetário (lógica paradoxal) para evitar insustentabilidade financeira pelo foco exclusivo em arte e desinteresse estético perante a exploração monetária (Davel \& Cora, 2016). A estética explica como alianças ou pontes são feitas, gerando alternativas para a dicotomia posta.

- O caráter intersubjetivo, coletivo, memorial, sensorial, estético (gosto, vínculos e referências), cultural, humano, local e material em uma co-criação de valor (Elias et al., 2018), distribuição e consumo entre artistas, críticos, consumidores, produtores, empresários, intermediários e comunidades (Gagliardi, 2009; Klamer, 2011; Khaire, 2015, 2017).

- O caráter estético da criatividade e da inovação e sua relação com a legitimação, o que se relaciona ao gosto, julgamento, aceitação (Dalpiaz, Rindova \& Ravasi, 2016; Frankelius, 2011; Islam, Endrissat \& Noppeney, 2016; Lingo \& O’Mohany, 2010) e a mudança. Isto envolve códigos semióticos e de base material (uma conjunção estética), cruciais no contexto das indústrias criativas (Jones, Lorenzen \& Sapsed, 2015).

- As ontoepistemologias e metodologias positivistas e funcionalistas tradicionais do empreendedorismo (Davel \& Cora, 2016; Elias et al, 2018), contrastantes com a natureza estética das artes e cultura (Beckman, 2015) que requerem a consideração plena da subjetividade e do interpretacionismo (Gherardi, 2019; Linstead \& Hopfl, 2000; Strati, 2007; Wood Jr. \& Csillag, 2001).

- As complexidades e peculiaridades estéticas das lógicas artística, cultural e arriscada, permitindo entender e aprofundar tanto diferentes lógicas artísticas, culturais e arriscadas, quanto problematizar aspectos relacionados, por exemplo, à críticas sobre alienação.

- A complexidade da estética na contribuição à temáticas como educação (Bryan, \& Harris, 2015), perfil, organização e gestão, política, singularidades, tecnologias, trabalho e carreira.

- A relação com tempo e a prática de carreira a partir dos parâmetros de excelência profissional nas artes (Kaufman \& Sternberg, 2015).

- A necessidade da estética na mitigação de aspectos críticos, reduzindo impactos negativos (Bryan \& Harris, 2015).

Outro tipo de carência decorre de um desafio metodológico. As metodologias apontam estudos de caso (principalmente para desvendar singularidades), entrevistas, levantamentos, métodos mistos e revisões de literatura (Hausmann \& Heinze, 2016). Observamos levantamentos longitudinais em técnicas de análise quantitativa, crescimento de metodologias interpretacionistas, observação participante, análises de discurso, semiótica, havendo alguma adesão a usos audiovisuais, história oral, análises com alguma relação com a arte, como as análises estéticas em diferentes manifestações artísticas. Estes estudos representam outras lógicas de atuação, mas observamos carência de metodologias refinadas à análise da sensualidade, prazer, gosto, encantamento, dispondo-se contra o individualismo metodológico e economicismo do empreendedorismo (Cunningham \& Potts, 2015; Steyaert, 2007) para compreender a teorização sensível da complexidade do campo. O desafio encontra-se na abertura metodológica com base nas artes e nas humanidades para gerar melhores análises estéticas, humanas, subjetivas, complexas, sensíveis, heurísticas, elusivas, experienciais e tácitas.

\section{Por uma via de renovação: Estética como perspectiva de pesquisa}


Entender o empreendedorismo nas artes e na cultura a partir da estética torna-se estratégico para o avanço da pesquisa futura por, no mínimo, três razões: (a) a estética é o principal valor a ser perseguido pelo empreendedorismo e isto é reforçado pelas relações estéticas já destacadas nas pesquisas sobre economia criativa (Koivunen \& Rehn, 2009; Jones, Lorenzen \& Sapsed, 2015); (b) a estética norteia todos os processos de criação, inovação, produção e consumo; e (c) a estética fundamenta as principais lógicas, temáticas e impactos do campo. Tanto o empreendedorismo artístico-cultural como a estética buscam o sensível, a beleza, as emoções, os sentimentos e significados cruciais e complexos nos campos das artes e cultura. Isto eleva a importância da estética de um tipo de aspecto popularmente conhecido na contemporaneidade como consumo para um conhecimento complexo e rico em discussões filosóficas. Apesar das muitas e conflitantes definições, a estética enquanto campo moderno de conhecimento (Strati, 2007; Taylor \& Hansen, 2005) busca, dentre outras coisas, compreender a assimilação de valores subjetivos em obras, produtos ou organizações.

O conhecimento estético é complexo e extenso, originando-se em tradições filosóficas, hermenêuticas, fenomenológicas e artísticas (Gaut \& Lopes, 2013; Strati, 2019). Em síntese, a estética remete às percepções de nosso corpo, por meio dos órgãos dos sentidos (visão, audição, tato, olfato e paladar), que interpretam o mundo em juízos de gosto e percepções sobre o que é belo, feio, pitoresco, sagrado, sublime, cômico, etc. (Strati, 2007). Estes juízos denominam-se de categorias estéticas (Strati, 2007). Assim, ao dizermos que algo é estético (etimologicamente, aisth vem do verbo grego sentir), expressamos que este algo é sensível e/ou, por exemplo, belo. Como essa sensibilidade e julgamentos são particulares, este algo é também distinto, memorável, auto referencial. Por isso, o conhecimento gerado pelos estudos em estética é um conhecimento alternativo ao conhecimento lógico, objetivo, racional e cognitivo (Strati, 2007).

No campo da Administração e dos Estudos organizacionais, este conhecimento denomina-se de estética organizacional (Strati, 2007; Taylor \& Hansen, 2005; Wood Jr. \& Csillag, 2001; Linstead \& Hopfl, 2000). Trata-se de um campo que estuda nossas percepções estéticas na vida das organizações (Strati, 2019). Suas unidades de análise envolvem o estudo das sensibilidades e percepções pessoais e interações empáticas coletivas pela expressividade do corpo (corporeidade), categorias estéticas, gostos, conhecimento tácito e prático, a relação entre sujeitos, mundo e objetos por sua característica intersubjetiva, enraizamentos, trocas e mudanças culturais, considerando fatores históricos e contexto (Strati, 2007; 2019; Gherardi, 2019). O foco recai em como os sujeitos empatizam ou criam sentimentos de pertença e vínculo a pessoas, comunidades, coisas e grupos por gosto (Gherardi, 2019).

\subsection{Relevância da estética organizacional na pesquisa do empreendedorismo na arte e na cultura}

A relevância estética na pesquisa do empreendedorismo artístico e cultural se faz tanto de forma micro, desvelando subjetividades do fazer empreendedor, quanto meso e macro, como a forma com que estas subjetividades interferem no cotidiano organizacional, educacional, político, tecnológico, social, cultural, artístico e econômico. Algumas pesquisas já apontam fatores ou problemas estéticos no fazer empreendedor sem compreendê-los como estéticos (Wilson \& Stokes, 2002). Outras pesquisas reconhecem a estética ao falar de co-criação de ideias em processos ambíguos de emergência e qualidade estética (Lingo \& O'Mohany, 2010), na relação entre formas de arte e traumas culturais (Santoro, 2006); normas, padrões, hierarquias e fronteiras estéticas em arte (Fleming \& Roses, 2007), mobilidade estética ou cultural (Pedroni \& Volanté, 2014). Algumas pesquisas reconhecem o poder simbólico e a influência das artes para resolver problemas na incorporação de valores e significados em produtos tradicionais (Àrtico \& Tama, 2018). Porém, encontramos apenas uma pesquisa que enfoca a estética do empreendedorismo nas artes, por meio do estudo da co-criação de valor estético entre empreendedores artísticos e seus clientes (Elias et al., 2018). Além disso, a pesquisa estética no campo do empreendedorismo para além das artes e cultura permanece limitada (Hjorth \& Steyaert, 2009; Mauksch, 2016; Poldner, Shrivastava, \& Branzei, 2017; Pritchard, Davey, \& Cooper, 2019; Smith, 2015; Vogt, 
Bulgacov, \& Elias, 2019; Warren \& Anderson, 2009; Willerding, 2015). Com base nas artes, solicita-se uma mudança e um novo paradigma estético para a atividade empreendedora (Bryan \& Harrys, 2015).

Estudar o empreendedorismo na cultura e nas artes pela perspectiva estética é relevante para revelar dimensões estéticas subjacentes à prática do fazer artístico-cultural: (a) entender a formação de sentimentos, emoções, gosto, perfis, beleza e demais julgamentos estéticos na aprendizagem, constituição e avaliação do empreendedorismo no tempo, (b) compreender a constituição formal e informal de cadeias, redes e fluxos de valor entre os diversos agentes (artistas, fãs, crítica, organizações, intermediários, fornecedores, comunidades locais e comunidades de gosto), (c) equilibrar valor estético, monetário e demais paradoxos, (d) entender a formação de signos, significados e referências culturais, (e) apreender aspectos mercadológicos (competição, sensibilidades de consumo, confiabilidade do mercado, variações de gosto e estilo, sucesso e fracasso), (f) revelar a importância de objetos e artefatos (instrumentos, tecnologias, desmistificar obras), (g) destacar a criação de invenções e inovações culturais e estéticas (critérios de originalidade e imitação estética), (h) enfatizar questões de poder e hierarquia estética, (i) entender formas de construção, transmissão e preservação de valores, (j) desvendar o gosto e prazer na busca do incerto, imaginação estética, $(\mathrm{k})$ refletir sobre a aderência de currículos educacionais e políticas às realidades artísticas locais, (l) entender a estruturação e gestão estética de organizações, (m) desvendar as tecnologias, capital humano de tecnologias e seus impactos tácitos, (n) caracterizar as especificidades e necessidades estéticas de trabalho e carreira e (o) compreender as formas de lidar esteticamente com impactos culturais, sociais, políticos, econômicos, artísticos, em suma, os transbordamentos estéticos.

A estética organizacional torna-se uma perspectiva promissora e relevante para abrir novos caminhos de pesquisa. Ela contribui para compreender aspectos de formação, transmissão, diferenciação, classificação, enquadramento, avaliação, identidade, escolha, definição e formas de imposição social de valores estéticos. Também estimula a compreensão sobre as visões do empreendedorismo como agente de subversão ao economicismo (geração de valores subjetivos) e/ou agente de aderência ao economicismo (geração de lucros) na arte.

\section{Discussões}

Objetivando consolidar uma compreensão integrada da produção acadêmica sobre a relação entre empreendedorismo, cultura e arte e propor perspectivas renovadoras para pesquisas futuras, estruturamos várias categorias que permitem melhor entender singularidades, temáticas, impactos, forças e desafios desta produção. Além disso, destacamos e discutimos como a estética se torna uma perspectiva promissora e relevante para renovar a pesquisa sobre empreendedorismo cultural e artístico.

Ao falarmos de singularidades, elas foram apresentadas associadas à obra empreendedora (valores e consumo estéticos, inovação intersubjetiva) e ao processo empreendedor (lógicas artística, cultural, paradoxal e arriscada). Apesar de valores, consumo e inovação permearem processos, eles são desvelados na obra, motivada pelos processos descritos. Estas singularidades são destacadas pela diversidade e alcance de impactos do campo. Para além destas proposições, os resultados da pesquisa estimulam uma discussão em torno de alguns tipos de implicações: a) teórico-ontoepistêmicas; b) teórico-conceituais, c) metodológicas, d) práticos.

Em termos de implicações teórico-ontoepistêmicas, entendemos que existem ontoloepistemologias empreendedoras relacionais e processuais que valorizam o conhecimento tácito, coletivo, experiencial, situado, profundo, humano, estético, bem como o enfoque na prática (Hjorth \& Steyaert, 2009; Steyaert, 2007), promovido timidamente no contexto do empreendedorismo cultural (Marins \& Davel, 2019), mas que ainda requer muitos aprofundamentos. Os resultados desta pesquisa estimulam estudos futuros a considerarem esse aspecto, dentre outros fatores, pelo teor performativo (Gehman \& Soublière, 2017; Hadida, 2015) e prático (Hausmann \& Heinze, 2016). Isto contribui para evitar olhares e preocupações tradicionais e individualistas do empreendedorismo focadas no agente ou em perfis (Gartner, 1988), 
conforme preocupação apontada nas seção sobre temáticas e tendo em vista a oportunidade que o campo artístico oferta em termos de tensões diversas. São desafios em que a epistemologia da prática pode ajudar a reduzir romantizações em torno destas formas de tensões muitas focadas no indivíduo enquanto unidade de análise. Além disso, reforçamos a relação entre empreendedorismo e inovação, o que nos leva, em específico, ao desafio da busca histórica no mundo inovador da arte de sua relação com o empreendedorismo prático e cotidiano. O que há de cotidiano na inovação?

As implicações teórico-conceituais problematizam as distinções e comunalidades entre entendimentos sobre formas de empreendedorismo (cultural, artístico, criativo) e nestas, tendências à novas singularizações (empreendedorismo musical, teatral, etc.) tendo em vista a maior estetização do campo. $\mathrm{O}$ que é compartilhado? Terminologias trazem novos significados e carregam polissemias. $\mathrm{O}$ adjetivo cultural atrelado ao empreendedorismo pode afastá-lo do campo das artes (Lounsbury \& Glynn, 2001). $\mathrm{O}$ adjetivo artístico pode individualizar na perspectiva da agência. $\mathrm{O}$ adjetivo criativo pode generalizar, esvaziar e enfatizar as dimensões econômicas. Por outro lado, não indicamos a fuga de terminologias (usar apenas empreendedorismo), porque as singularidades repercutem em diferenças fundamentais conforme apontamos neste estudo. Ao identificarmos e discriminarmos as lógicas artística e cultural destacamos diferenças (como a lógica artística que tende à inovação e a lógica cultural que tende à preservação) e com isso, abrimos oportunidades para identificar níveis de conflito e possibilidades de conciliação internas, sendo curioso como isto anima, por exemplo, processos de inovação. Em adição, as singularidades são permeadas por forças entre o individual e o coletivo, mas indicam um caminho em direção ao coletivo. Neste sentido, reforçamos a relevância de aplicações transdisciplinares, a adoção de visões críticas e principalmente o enfoque na perspectiva da estética organizacional de forma a contribuir com a reflexão e construção teórica sobre estas problematizações.

Como implicações metodológicas, os resultados da pesquisa chamam a atenção para a importância de abordagens metodológicas que busquem o aprofundamento sensível, como por exemplo, a mobilização de etnografias (como a chamada etnografia estética) (Marins \& Davel, 2020; Warren, 2008), e de unidades de análise pautadas na prática (Gagliardi, 1999; Gherardi, 2019; Reckwitz, 2017; Strati, 2019) por seu potencial integrador. Isto potencializa análises sensíveis e críticas, necessárias ao campo.

As implicações práticas envolvem o aprimoramento de campos. Ao falarmos da educação, pode-se explorar dinâmicas para a educação empreendedora camufladas em cursos artísticos ou mesmo no trabalho artístico, extrapolando o já escasso mundo da educação empreendedora nas artes e cultura (focado na graduação e pós graduação), se estendendo para formações de base. Isto ainda contribui com métodos inovadores em currículos e aproximação da realidade de estudantes, sendo mais focados em arte e transformação. O que permite um melhor entendimento sobre o que singulariza a prática dos empreendedores culturais e artísticos, os ajuda a melhor praticar. Com isso, estamos na rota reflexiva de potencializar o desenvolvimento cultural e artístico nos territórios em que a economia criativa é crucial. Ao falarmos de política, as implicações nos levam a fortalecer ações de estímulo, regulação e avaliação coerentes com as peculiaridades e necessidades do fazer empreendedor no campo das artes e da cultura. Para empreendedores e empreendedoras as implicações levam a formas mais rápidas, eficazes e democráticas de conhecimento, reduzindo sofrimentos, custos e percalços que individualizam excessivamente trajetórias.

\section{Conclusões}

Elaboramos um levantamento sistemático que gerou algumas contribuições para o avanço do conhecimento sobre o empreendedorismo nas artes e na cultura: (a) estruturação e categorização de singularidades (valor, inovação e consumo subjetivos, lógicas artística, cultural, paradoxal e arriscada), temáticas, impactos (econômicos, sociais, culturais, artísticos e políticos, diretos, indiretos, positivos e negativos), forças e desafios, e (b) proposta e discussão da estética organizacional como perspectiva teórico-conceitual para renovar pesquisas futuras. 
No processo de pesquisa, três aspectos revelam limitações a serem consideradas. O primeiro aspecto é que, sendo os termos de busca relacionados ao empreendedorismo, um aporte teórico recente e criticamente tensionado no campo das artes e da cultura (campos que vem, por sua vez, de discussões teóricas em torno da indústria cultural e economia da cultura), há aporte teórico extenso e com afinidades à temática, mas que não faz associações com termos como empreendedorismo, empreendedor, empreendedorismo cultural, criativo ou artístico. Alguns deles apareceram e foram considerados em nosso estudo quando foram associados ao empreendedorismo por outros autores trazidos em sua busca por explicações, além da importância na influência de constructos apresentados. Um exemplo desta associação se dá por termos como "trabalho/trabalhadores culturais ou da cultura", "trabalho artístico", "trabalho/trabalhadores criativos". Isto nos leva ao fato de que releituras estão sendo feitas na busca da identificação de empreendedorismo cultural e artístico no campo. O segundo aspecto é o viés das análises, já que a maior parte dos estudos é internacional (o que se trata de uma característica do campo na atualidade) e que, quando trazem fundamentos empíricos, trazem a arte e a cultura por estéticas internacionais. Isto nos leva a estimular a investigação do empreendedorismo e suas singularidades artísticas e culturais locais, o que outras lentes, como a estética, vem a contribuir. O terceiro aspecto é a necessidade de aprofundamento nas temáticas nos campos das artes, de forma a enriquecer, por exemplo, a reflexão sobre as lógicas, como a artística.

\section{Referências}

Araújo, G. F. de, \& Davel, E. P. B. (2018). Educação empreendedora, experiência e John Dewey. Revista Pensamento Contemporâneo em Administração, 12(4), 1-16. https://doi.org/10.12712/rpca.v12i4.13291

Àrtico, C. I., \& Tamma, M. (2018). Culture-Based Products: Integrating Cultural and Commercial Strategies. In Innerhofer E., Pechlaner H., \& Borin E. (Eds.) Entrepreneurship in Culture and Creative Industries. FGF Studies in Small Business and Entrepreneurship. (11-22). Cham: Springer. https://doi.org/10.1007/978-3-319-65506-2 2

Banks, M., Gill, R., \& Taylor, S. (2014). Theorizing Cultural Work: Labour, Continuity and Change in the Cultural and Creative Industries. London: Routledge.

Banks, M., Lovatt, A., Connor, J. O., \& Raffo, C. (2000). Risk and trust in the cultural industries. Geoforum, 31(4), 453-464.

Barry, D. (2011). Art and Entrepreneurship, apart and together. In Scherdin, M., \& Zander, I. (Eds.). Art entrepreneurship. (154-168). Cheltenham: Edward Elgar.

Becker, H. (1982). Art Worlds. Berkeley: University of California Press.

Becker, S. G. (1964). Human Capital. New York: Columbia University Press.

Beckman, G. D (2015). Entrepreneuring the Aesthetic: arts entrepreneurship and reconciliation. In Baker, T., \& Welter, F. The Routledge Companion to Entrepreneurship. London: Routledge

Beckman, G. D., \& Essig, L. (2012). Arts entrepreneurship: a conversation. Artivate: A Journal of Entrepreneurship in the Arts, 1(1), 1-8.

Beuys, J. (2004). Whats is Art? Conversation with Joseph Beuys. New York: Clairview Books.

Birnkraut, G. (2018). The New Socio-Cultural Entrepreneurs: At the Crossroads Between Social Enterprises and Arts. In Innerhofer E., Pechlaner H., \& Borin E. (Eds.), Entrepreneurship in Culture and Creative Industries: Perspectives from Companies and Region. (171-182). Cham: Springer. https://doi.org/10.1007/978-3-319-65506-2 9

Borghoff, B. (2018). Entrepreneurial Storytelling as Narrative Practice in Project and Organizational Development. In Innerhofer, E., Pechlaner, H., \& Borin, E. (Eds.) Entrepreneurship in Culture and Creative Industries: Perspectives from Companies and Regions, (63-84). Cham: Springer. https://doi.org/10.1007/978-3-319-65506-2 5

Borin, E., Donato, F., \& Sinapi, C. Financial Sustainability of Small- and Medium-Sized Enterprises in the Cultural and Creative Sector: The Role of Funding. In Innerhofer, E., Pechlaner, H., \& Borin, E. (Eds.) Entrepreneurship in Culture and Creative Industries: Perspectives from Companies and Regions. (45-62) Cham: Springer. https://doi.org/10.1007/978-3-319-65506-2_4

Bourdieu, P. (1989). O poder simbólico. Rio de Janeiro: Bertrand Brasil.

Bourdieu, P. (1993). The Field of Cultural Production. Essays on Art and Literature. New York: Columbia University Press. 
Bourdieu, P. (1996). As Regras da Arte: Gênese e Estrutura do Campo Literário. São Paulo: Companhia das Letras. Bourdieu, P . (2007). A Distinção: crítica social do julgamento. Sao Paulo: Edusp.

Brandenburg, S., Roosen, T., \& Veenstra, M. (2016). Toward an adapted business modeling method to improve entrepreneurial skills among art students. Artivate: A Journal of Entrepreneurship in the Arts, 5(1), 25-33.

Bridgstock, R. (2012). Not a dirty word: Arts entrepreneurship and higher education. Arts \& Humanities in Higher Education, 12(2-3), 122-137. https://doi.org/10.1177/1474022212465725

Bryan, T; \& Harrys, D. (2015). The Aesthetic Value Exchange: A Potential Framework for the Arts Entrepreneurship Classroom. Jaee. Journal of Arts Entrepreneurship Education, 1(1), 25-54.

Bujor, A., \& Avasilcai, S. (2016). The Creative Entrepreneur: A Framework of Analysis. Procedia - Social and Behavioral Sciences, 221, 21-28. https://doi.org/10.1016/j.sbspro.2016.05.086

Campos, I. M., \& Davel, E. (2017). Identidade, arte e gestão em prol do empreendedorismo cultural: sarau empreendedor como tecnologia social. RACE - Revista de Administração, Contabilidade e Economia, 16(2), 783-808. https://doi.org/10.18593/race.v16i2.13270

Campos, I. M., \& Davel, E. (2018). Empreendedorismo Cultural, Aprendizagem e Identidade Territorial: O Desbravamento de Jovens Músicos do Nordeste de Amaralina. Administração Pública e Gestão Social, 9, 66-73. https://doi.org/10.21118/apgs.v10i1.1585

Cattani, C., Ferriani, S., \& Colucci, M. (2015). Creativity in Social Networks: A Core-Periphery Perspective. In Jones, C., Lorenzen, M., \& Sapsed, J. (Eds.) The Oxford Handbook of Creative Industries (75-95). United Kingdom: Oxford University Press.

Caves, R. E. (2000). Creative Industries: Contracts Between Art and Commerce. Cambridge: Harvard University Press.

Chang, W. J., \& Wyszomirski, M. (2015). What is Arts Entrepreneurship? Tracking the Development of its Definition in Scholarly Journals. A Journal of Entrepreneurship in the Arts, 4(2), 11-31.

Cora, M. A. J. (2016). Empreendedores Criativos: Uma Análise sobre o Trabalho na Cultura. Rigs: Revista Interdisciplinar de Gestão Social, 5(2), 71-89.

Cunningham, S., \& Potts, J (2015). Creative Industries and the Wider Economy. In Jones, C., Lorenzen, M., \& Sapsed, J. (Eds.) The Oxford Handbook of Creative Industries (387-404). United Kingdom: Oxford University Press.

Currid-Halkett, E. (2015). Star and Stardom in the Creative Industries. In Jones, C., Lorenzen, M., \& Sapsed, J. (Eds.) The Oxford Handbook of Creative Industries (171- 185). United Kingdom: Oxford University Press.

Dalpiaz, E., Rindova, V., \& Ravasi, D. (2016). Combining Logics to Transform Organizational Agency: Blending Industry and Art at Alessi. Administrative Science Quarterly, 61(3), 1-46. https://doi.org/10.1177/0001839216636103 Davel, E., \& Cora, M. A. J. (2016). Empreendedorismo cultural: cultura como discurso, criação e consumo simbólico. Politicas Culturais Em Revista, 9(1), 363-397. http://dx.doi.org/10.9771/pcr.v9i1.10035.

Davies, R., \& Sigthorsson, G. (2013). Introducing the Creative Industries: From Theory to Practice. Los Angeles: Sage Publications.

Debenedetti, S., \& Larceneux, F. (2011). "The Taste of Others": Divergences in Tastes between Professional Experts and Ordinary Consumers of Movies in France. Recherche et Applications en Marketing, 26(4), 71-88. https://doi.org/10.1177/205157071102600404

DiMaggio, P. (1982). Cultural Entrepreneurship in Nineteenth Century Boston: Tthe Creation of an Organization Base for High Culture in America. Media, Culture and Society, 4(1), 33-50.

Eikhof, D. R., \& Haunschild, A. (2006). Lifestyle Meets Market: Bohemian Entrepreneurs in Creative Industries. Creativity and Innovation Management, 15(3), 234-241. https://doi.org/10.1111/j.1467-8691.2006.00392.x

Eikhof, D. R., \& Haunschild, A. (2007). For art's sake! Artistic and economic logics in creative production. Journal of Organizational Behavior, 28(5), 523-538. https://doi.org/10.1002/job

Elias, S. R. S. T. A., Chiles, T. H., Duncan, C. M., \& Vultee, D. M. (2018). The Aesthetics of Entrepreneurship: How Arts Entrepreneurs and their Customers Co-create Aesthetic Value. Organization Studies, 39(2-3), 345-372. https://doi.org/10.1177/0170840617717548

Ellmeier, A. (2003). Cultural Enterpreneurialism: on the changing relationship between the arts, culture and employment. International Journal of Cultural Policy, 9(1), 1-15. https:// doi.org/10.1080/1028663032000069158 
Essig, L. (2015). Means and Ends: A Theory Framework for Understanding Entrepreneurship in the US Arts and Culture Sector. Journal of Arts Management Law and Society, 45(4), 227-246. https://doi.org/10.1080/10632921.2015.1103673

Essig, L. (2017). Same or Different? The "Cultural Entrepreneurship" and "Arts Entrepreneurship" Constructs in European and US Higher Education. Cultural Trends, 26(2), 125-137. https://doi.org/10.1080/09548963.2017.1323842

Ettinger, V. M. T. de M. (2015). Líderes empreendedoras do Pacífico Colombiano: cultura, identidade e associativismo. Revista Interdisciplinar de Gestão Social, 4(2), 43-67.

Florida, R. L. (2012). The rise of the creative class, revisited. New York: Basic Books.

Formica, P. (2020). Business, Innovation and the Arts: The Golden Encounter. In Innovation and the Arts: The Value of Humanities Studies for Business (1-42). New York: Emerald Publishing Limited. https://doi.org/10.1108/978-178973-885-820201001

Frankelius, P. (2011). Innovation Processes: experience drawn from the creation of Dalhala. In Scherdin, M.; Zander, I. (Eds.). Art Entrepreneurship. (98-141). Cheltenham: Edward Elgar.

Gagliardi, P. (2006). Exploring the Aesthetic Side of Organizational Life. In Clegg, S. R., Hardy, C., Lawrence, T. B. \& Nord, W. R. (Eds.), The Sage Handbook of Organization Studies (701-724). London: Sage Publications. https://doi.org/http://dx.doi.org/10.4135/9781848608030.n25

Gartner, W. B. (1989). "Who is an entrepreneur?" is the wrong question. Entrepreneurship Theory and Practice, 13(4), 47-68.

Gaut, B., \& Lopes, D. M. (2013). The Routlegde Companion to Aesthetic. $3^{a}$ ed. London: Routledge.

Gehman, J., \& Soublière, J. F. (2017). Cultural entrepreneurship: from making culture to cultural making. Innovation: Management, Policy and Practice, 19(1), 61-73. https://doi.org/10.1080/14479338.2016.1268521

Gherardi, S. (2019). How to Conduct a Practice-Based Study: Problems and Methods (2a ed.). Cheltenham: Edward Elgar. https://doi.org/10.4337/9781788973564

Gillespie, C. H. (2007). Gastrosophy and Nouvelle Cuisine: Entrepreneurial Fashion and. British Food Journal, 96(10), 19-23. https://doi.org/https://doi.org/10.1108/00070709410072472

Guerra, J. R. F. (2015). O Filme ao Redor: O Empreendedorismo Cultural na Produça de Cinema em Pernambuco. Tese de Doutorado. Universidade Federal de Pernambuco. Brasil. Disponível: https://repositorio.ufpe.br/handle/123456789/29401.

Guerra, J. R. F., \& Paiva Júnior, F. G. (2011). Cultural Entrepreneurship in Film Production: Revien of Administration and Innovation - RAI, 8(3), 78-99. https://doi.org/10.5773/rai.v8i3.792

Guo, W., \& Wyszomirski, M. J. (2018). Arts Entrepreneurship in China: Exploring the Professional Career Development Model for Chinese Emerging Western Classical Musicians. The Journal of Arts Management, Law, and Society, 49(3), 1-15. https://doi.org/10.1080/10632921.2018.1523764

Hadida, A. L. (2015). Performance in the Creative Industries. In Jones, C., Lorenzen, M., \& Sapsed, J. (eds.). The Oxford Handbook of Creative Industries (219-250). Oxford: Oxford University Press.

Hagoort, G. (2007). Cultural Entrepreneurship. Utrecht: Utrecht University School of the Arts.

Hagoort, G., Thomassen, A., \& Kooyman, R. (2012). Pioneering Minds Worldwide: On the Entrepreneurial Principles of the Cultural and Creative Industries. Delft: Eburon Publishers.

Hartley, J., Wen, W., \& Li, H. S. (2015). Creative Economy and Culture: Challenges, Changes and Futures for the Creative Industries. London: Sage Publications.

Hausmann, A. (2010). German artists between bohemian idealism and entrepreneurial dynamics: Reflections on cultural entrepreneurship and the need for start-up management. International Journal of Arts Management, 12(2), 1729.

Hausmann, A., \& Heinze, A. (2016). Entrepreneurship in the cultural and creative industries: insights from an emergent field. Artivate: A Journal of Entrepreneurship in the Arts, 5(2), 7-22.

Hesmondhalgh, D, \& Baker, S (2011). Creative labour: media work in three cultural industries. London: Routledge.

Hirsch, P. M. (1972). Processing Fads and Fashions: An Organization-Set Analysis of Cultural Industry Systems. American Journal of Sociology, 77(4), 639-659. https://doi.org/10.4324/9780203993026

Hjorth, D., \& Steyaert, C. (2009). Politics and Aesthetics of Entrepreneurship. Cheltenham: Edward Elgar Pub. 
Holt, D., \& Cameron, D. (2010). Cultural Strategy: Using Innovative Ideologies to Build Breakthrough Brands. Oxford: Oxford University Press.

Howkins, J. (2001). The Creative Economy: How People Make Money from Ideas. London: Penguin Books.

Hoyte, C. (2019). Artisan Entrepreneurship: A Question of Personality Structure? International Journal of Entrepreneurial Behavior \& Research, 25(4), 615-632. https://doi.org/10.1108/IJEBR-02-2018-0099

Islam, G., Endrissat, N., \& Noppeney, C. (2016). Beyond 'the Eye' of the Beholder: Scent Innovation Through Analogical Reconfiguration. Organization Studies, 37(6), 1-27. https://doi.org/10.1177/0170840615622064

Jones, C., Lorenzen, M., \& Sapsed, J. (2015). Creative Industries: A Typology of Change. In: Jones, C., Lorenzen, M., \& Sapsed, J. (eds.). The Oxford Handbook of Creative Industries. (3-31). Oxford: Oxford University Press.

Judice, V. M. M., \& Furtado, S. C. (2014). Gestão de empreendimentos criativos e culturais: estudo de uma organização de ensino e difusão de ritmos afro-brasileiros em São João Del-Rei (MG). Organizações \& Sociedade, 21(69), 293-314. https:// doi.org/10.1590/S1984-92302014000200006

Kaufman, J. C. \& Sternberg, R. J. (2015). The Creative Mind. In Jones, C., Lorenzen, M., \& Sapsed, J. (eds.) The Oxford Handbook of Creative Industries. (33-49). Oxford: Oxford University Press.

Kavousy, E., Shahosseini, A., Kiasi, S., \& Ardahaey, F. (2010). Cultural entrepreneurship strategies in Iran. Serbian Journal of Management, 5(2), 227-241.

Khaire, M. (2015). Entrepreneurship in Creative Industries and Cultural Change: Art, Fashion and Modernity in India. In: Jones, C., Lorenzen, M., \& Sapsed, J. (eds.) The Oxford Handbook of Creative Industries. (200-218). Oxford: Oxford University Press.

Khaire, M. (2017). Culture and Commerce: The V alue of Entrepreneurship in Creative Industries. Stanford: Stanford Business Books.

Klamer, A. (2010). Doing the Right Thing: A V alue Based Economy. London: Ubiquity Press.

Klamer, A. (2011). Cultural entrepreneurship. Review of Austrian Economics, 24(2), 141-156. https://doi.org/10.1007/s11138-011-0144-6

Koivunen, N., \& Rehn, A. (2009). Introduction. In Koivunen, N., \& Rehn, A. (Eds.) Creativity and the contemporary economy. (7-12). Copenhagen: Copenhagen Business School Press.

Koivunen, N., \& Wennes, G. (2011). Show us the sound! Aesthetic leadership of symphony orchestra conductors. Leadership, 7(1), 51-71. https://doi.org/10.1177/1742715010386865

Kolb, B. M. (2015). EntrepreneurshipfFor the Creative and Cultural Industries. London: Routledge.

Kolsteeg, J. (2013). Situated Cultural Entrepreneurship. Artivate: A Journal of Entrepreneurship in the Arts, 2(1), 3-13.

Konrad, E. D. (2013). Effects of Network Ties in the Culture Business. In: Pechlaner, H., Abfalter, D. and Dal Bò, G. (Eds.), Cultural Entrepreneurship - Challenges and Strategies. (147-62). Bolzano: EURAC

Korzen, K. (2015). Arts Entrepreneurship in Higher Education: Preliminary Inventories and Examinations. Jaee. Journal of Arts Entrepreneurship Education, 1(1), 24-54. https://doi.org/10.46776/jaee.v1.31

Kuznetsova-Bogdanovits, K., Ranczakowska-Ljutjuk, A. \& Kiitsak-Prikk, K (2018). Entrepreneurial Education in Arts Universities: Facilitating the Change to the Entrepreneurial Mindset. In: Innerhofer, E., Pechlaner, H., \& Borin, E. (Eds.) Entrepreneurship in Culture and Creative Industries: Perspectives from Companies and Regions. (157-170). Cham: Springer https://doi.org/10.1007/978-3-319-65506-2_8

Laan, E. Van Der, \& Kuipers, G. (2016). How aesthetic logics shape a cultural field: Differentiation and consolidation in the transnational field of fashion image, 1982 - 2011. Poetics, 56, 64-84. https://doi.org/10.1016/j.poetic.2016.01.001

Lange, B. (2018). The Paradox Between Individual Professionalization and Dependence on Social Contexts and Professional Scenes. In: Innerhofer, E., Pechlaner, H., \& Borin, E. (Eds.) Entrepreneurship in Culture and Creative Industries: Perspectives from Companies and Regions. (85-98). Cham: Springer https://doi.org/10.1007/978-3-319$\underline{6506-2} 6$

Lawrence, T. B., \& Phillips, N. (2002). Understanding Cultural Industries. Journal of Management Inquiry, 11(4), 430441. https://doi.org/10.1177/1056492602238852.

Leadbeater, C., \& Oakley, K. (1999). The Independents: Britain's New Cultural Entrepreneur. London: Demos.

Lena, J. C., \& Pachucki, M. C. (2013). The sincerest form of flattery: Innovation, repetition, and status in an art movement. Poetics, 41(3), 236-264. https://doi.org/10.1016/i.poetic.2013.02.002 
Lindkvist, L., \& Hjorth, D. (2015). Organizing Cultural Projects through Legitimising as Cultural Entrepreneurship. International Journal of Managing Projects in Business, 8(4), 696-714. https://doi.org/10.1108/IJMPB-07-2015-0064

Lindqvist, K. (2011). Artist Entrepreneurs. In Scherdin, M.; Zander, I. (Ed.). Art Entrepreneurship. (10-22). Cheltenham: Edward Elgar Publishing.

Lingo, E. L., \& O’ Mahony, S. (2010). Nexus Work: Brokerage on Creative Projects. Administrative Science Quarterly, 55(1), 47-81. https://doi.org/10.2189/asqu.2010.55.1.47

Lingo, E. L., \& Tepper, S. J. (2013). Looking Back, Looking Forward: Arts-Based Careers and Creative Work. Work and Occupations, 40(4), 337-363. https://doi.org/10.1177/0730888413505229

Lounsbury, M., \& Glynn, M. A. (2001). Cultural entrepreneurship: Stories, legitimacy, and the acquisition of resources. Strategic Management Journal, 22(6-7), 545-564. https:// doi.org/10.1002/smj.188

Lounsbury, M., \& Glynn, M. A. (2019). Cultural Entrepreneurship: A new Agenda for the Study of Entrepreneurial Processes and Possibilities. Cambridge: Cambridge University Press.

Loy, A., \& AAgeson, T. (2018). Creative economy entrepreneurs: From Startup to Success. Santa Fé: Global Center for Cultural Entrepreneurship.

Marins, S. R., \& Davel, E. P. B. (2019). Empreendedorismo como Prática: Empreendedorismo Cultural na Prática Festiva do Pagode Baiano. Teoria e Prática em Administração, 9(2), 14-34. https://doi.org/http://dx.doi.org/10.21714/2238-104X2019v9i2-43231

Marins, S. R., \& Davel, E. P. B. (2020). Etnografia estética na pesquisa em estudos organizacionais: princípios, processos e desafios. Revista Brasileira de Estudos Organizacionais, 7(2), 1-39.

Marriott, L., \& Miller, A. (2012). Accounting for Cultural Well-Being: an Exploratory Study of New Zealand Regions. Pacific Accounting Revie, 24(2), 112-137. https://doi.org/10.1108/01140581211258443

Mauksch, S. (2016). Managing the dance of enchantment: An ethnography of social entrepreneurship events. Organization, 24(2), 2016.

McRobbie, A. (2011) 'Re-Thinking Creative Economy as Radical Social Enterprise'. Disponível: http://www.variant.org.uk/.

Meisiek, S., \& Haefliger, S. (2011). Invinting the Inexpected: Antrepreneurship and the Arts. In Scherdin, M.; Zander, I. (Ed.). Art Entrepreneurship. (78-97). Cheltenham: Edward Elgar.

Menger, P.-M. (2014). The Economics of Creativity: Arts and Acbievement under Uncertainty. Cambridge: Harvard University Press.

Merleau-Ponty, M. (1999) Fenomenologia da Percepção. $2^{a}$ ed. São Paulo: Martins Fontes.

Mitra, J. (2020). On Regarding the Value of Aesthetics for Entrepreneurship. In Formica, P. \& Edmondson, J. (Eds.) Innovation and the Arts: The V Value of Humanities Studies for Business (73-90). Bindley: Emerald Publishing Limited. https://doi.org/10.1108/978-1-78973-885-820201004

Montagnino, F. M. (2018). Beyond the 'Great Derangement': Will the Humanities Lead Ecological Transition? In Formica, P. \& Edmondson, J. (Eds.) Innovation and the Arts: The Value of Humanities Studies for Business (73-90). Bindley: Emerald Publishing Limited. https://doi.org/10.1108/978-1-78973-885-820201007

Morris, J. W. (2014). Artists as Entrepreneurs, Fans as Workers. Popular Music and Society, 37(3), 273-290. https://doi.org/10.1080/03007766.2013.778534

Morrow, G. (2018). Artist Management: Agility in the Creative and Cultural Industries. In Rentschler, R. (Ed.). Mastering management in the Creative and Cultural Industries. London: Routledge

Naudin, A. (2015). Cultural Entrepreneurship: Identity and Personal Agency in the Cultural Worker's Experience of Entrepreneurship. Tese de Doutorado. Warwick: University of Warwick.

Neff, G., Wissinger, E., \& Zukin, S. (2005). Entrepreneurial Labor among Cultural Producers: “Cool” Jobs in "Hot" Industries. Social Semiotics, 15(3), 307-334. https://doi.org/10.1080/10350330500310111

Oliveira, P. G. G., Ribeiro, R. A., Cabral, Augusto, C., \& Santos, S. M. (2016). Economia criativa e o empreendedorismo no Ceará: um estudo de campo em uma empresa de design. Revista Brasileira de Gestão e Inovação, 3(2), 110-126.

Papathanasiou-Zuhrt, D., Russo, A., \& Kutsikos, K. Experience-Driven Cultural Entrepreneurship: Business Models and Regional Development in the "World of Frederick II Hohenstaufen". In Innerhofer E., Pechlaner 
H., Borin E. (Eds.) Entrepreneurship in Culture and Creative Industries: Perspectives from Companies and Regions. (327-340). Cham: Springer. https://doi.org/10.1007/978-3-319-65506-2 18

Patten, T. (2016). "Creative?”, "Entrepreneur?” - Undersatinding The Creative Industries Entrepreneur. Arrivate: A Journal of Entrepreneurship in the Art, 5(2), 23-42.

Pechlaner, H. \& Innerhofer, E (2019). When Culture Meets Economy. In Innerhofer, E., Pechlaner, H., \& Borin, E. (Eds.) Entrepreneurship in Culture and Creative Industries: Perspectives from Companies and Regions, 37(4), 229-244.

Pedroni, M., \& Volonté, P. (2014). Art seen from outside: Non-artistic legitimation within the field of fashion design. Poetics, 43(1), 102-119. https://doi.org/10.1016/j.poetic.2014.01.007

Petrova, L. (2018). Cultural Entrepreneurship in the Context of Spillovers Within the Cultural and Creative Industries: The case of Design Practice for Social Change. In Innerhofer E., Pechlaner H., Borin E. (Eds) Entrepreneurship in Culture and Creative Industries. 197-211. Cham: Springer. https://doi.org/10.1007/978-3-31965506-2 11

Poldner, K., Shrivastava, P., \& Branzei, O. (2017). Embodied Multi-Discursivity: An Aesthetic Process Approach to Sustainable Entrepreneurship. Business and Society, 56(2), 214-252. https://doi.org/10.1177/0007650315576149 Poorsoltan, K. (2012). Artists as entrepreneurs. International Journal of Entrepreneurship, 16(1), 83-100.

Preece, S. B. (2011). Performing Arts Entrepreneurship: Toward a Research Agenda. The Journal of Arts Management, Law, and Society, 41(2), 103-120. https://doi.org/10.1080/10632921.2011.573445

Preece, S. B. (2015). Acquiring Start-Up Funding for New Arts Organizations. Nonprofit Management and Leadership, 25(4), 463-474. https:// doi.org/10.1002/nml.21131

Preece, S.B. (2014). Social Bricolage in Arts Entrepreneurship: Building a Jazz Society from Scratch. Artivate: A Journal of Entrepreneurship in the Arts, 3(1), 23-34

Pritchard, K.; Davey, K. M.; Cooper, H. (2019). Aesthetic labouring and the female entrepreneur: 'Entrepreneurship that wouldn't chip your nails'. International Small Business Journal, 37(4) 1-22. https://doi.org/10.1177/0266242618823408

Qian, H., \& Liu, S. (2018). Cultural Entrepreneurship in U.S. Cities. Journal of Urban Affairs, 40(8), 1-23. https://doi.org/10.1080/07352166.2018.1468223

Rae, D. (2005). Cultural Diffusion: A Formative Process in Creative Entrepreneurship? The International Journal of Entrepreneurship and Innovation, 6(3), 185-192. https://doi.org/10.5367/0000000054662764

Ravasi, D., \& Rindova, V. (2013). Criação de Valor Simbólico. Rigs: Revista Interdisciplinar de Gestão Social, 2(2), 13 35.

Ravasi, D., Rindova, V., \& Dalpiaz, E. (2012). The cultural side of value creation. Strategic Organization, 10(3), 231239. https://doi.org/10.1177/1476127012452824

Reckwitz, A (2017). How to Senses Organize the Social. In M. Jonas, \& L Beate. Praxeological Political Analysis. (pp. 56-66). New York: Routledge.

Reis, J. M., \& Zille, L. P. (2020). Empreendedorismo Cultural e Economia Criativa: a Companhia de Teatro "Grupo Galpão". Revista de Empreendedorismo e Gestão de Pequenas Empresas, 9(2), 97-122. https://doi.org/https://doi.org/10.14211/regepe.v9i2.1576

Rentschler, R. (2007). Painting equality: female artists as cultural entrepreneurial marketers. Equal Opportunities International, 26(7), 665-677. https://doi.org/10.1108/02610150710822302

Riot, E. (2013). Woman in Love, Artist or Entrepreneur? The Edifying, Mystifying Life of Coco Chanel. Society and Business Review, 8(3), 281-313. https://doi.org/10.1108/SBR-12-2012-0054

Rivetti, F., \& Migliaccio, M. (2018). Arts and Entrepreneurship: Disentangling the Literature. In Brito, S. M. (Ed.) Entrepreneurship: Trends and Challenges (p. 12). London: Intechopen. https://doi.org/10.5772/intechopen.72014

Ruiz, J., Colbert, F., \& Hinna, A. (2017). Arts and culture management. Academia Revista Latinoamericana de Administración, 30(2), 147-155. https://doi.org/10.1108/ARLA-02-2017-0032

Scherdin, M., \& Zander, I. (2011). Emerging themes and new research openings. In Scherdin, M.; Zander, I. (Ed.). Art Entrepreneurship. (169-186). Cheltenham: Edward Elgar Publishing.

Schiray, D. M., Carvalho, C. C., \& Afonso, R. (2017). Creative economy as a social technology approach. Academia Revista Latinoamericana de Administración, 30(4), 508-528. https://doi.org/10.1108/ARLA-06-2016-0160. https://doi.org/10.1108/arla-06-2016-0160. 
Scott, M. (2012). Entrepreneurs, Cultural entrepreneurship: Music Producers Mobilising and Converting Bourdieu's Alternative Capitals. Poetics, 40(3), 237-255. https://doi.org/10.1016/i.poetic.2012.03.002

Smith, R. (2015). Entrepreneurship and poetry: Analyzing an aesthetic dimension. Journal of Small Business and Enterprise Development, 22(3), 450-472. https://doi.org/10.1108/JSBED-09-2012-0103

Stefanovic, M. (2018). Creative Entrepreneurship in No Man's Land: Challenges and Prospects for a Metropolitan Area and Smaller Communities. Perspectives from the Never-Ending Transition. In Innerhofer E., Pechlaner H., Borin E. (Eds.) Entrepreneurship in Culture and Creative Industries: Perspectives from Companies and Regions. (311-325). Cham: Springer: https://doi.org/10.1007/978-3-319-65506-2_19

Steyaert, C. (2007). 'Entrepreneuring' as a conceptual attractor? A review of process theories in 20 years of entrepreneurship studies. Entrepreneurship and Regional Development, 19(6), 453-477. https://doi.org/10.1080/08985620701671759

Strati, A. (2007). Organização e Estética. Rio de Janeiro: FGV.

Strati, A. (2008). Do you do beautiful things? Aesthetics and art in qualitative methods of organization studies. In Buchanan, D., \& Bryman. A. (Eds.). The Sage Handbook of Organizational Research Methods, 230-245. London: Sage Publications. https://doi.org/10.13140/RG.2.1.1309.3849

Strati, A. (2019). Organizational Theory and Aesthetic Philosophies. New York: Routledge.

Svejenova, S., Pedersen, J. S., \& Vives, L. (2011). Projects of Passion: Lessons for Strategy from Temporary Art. In G. Cattani, S. Ferriani, L. Frederiksen, \& F. Taube (Eds.), Project-Based Organizing and Strategic Management (pp. 501-527). London: Emerald Group Publishing Limited.

Svejenova, S., Slavich, B., \& Sondo, G. A. (2015). Creative Entrepreneurs: The Business Models of Haute Cuisine Chefs. In C. Jones, M. Lorenzen, \& J. Sapsed (Eds.), The Oxford Handbook of Creative Industries. Oxford: Oxford University Press.

Swedberg, R. (2006). The Cultural Entrepreneur and the Creative Industries: Beginning in Vienna. Journal of Cultural Economics, 30(4), 243-261. https://doi.org/10.1007/s10824-006-9016-5

Taylor, S. S., \& Hansen, H. (2005). Finding form: Looking at the field of organizational aesthetics. Journal of Management Studies, 42(6), 1211-1231. https://doi.org/10.1111/j.1467-6486.2005.00539.x

Thom, M. (2014). The Suffering of Arts Entrepreneurs: Will Fine Art Students be Educated on How to Become Successfully Self-Employed? Journal of Education and Training Studies, 3(1), 64-77. https://doi.org/10.11114/jets.v3i1.587

Thom, M. (2015). The Entrepreneurial Value of Arts Incubators: Why Fine Artists Should Make Use of Professional arts Incubators. Artivate, 4(2), 33-56.

Thom, M. (2016). Crucial Skills for the Entrepreneurial Success of Fine Artists. Artivate: A Journal of Entrepreneurship in the Art, 5(1), 3-24.

Thom, M. (2017). The rough journey into arts entrepreneurship: Why arts entrepreneurship education is still in its infancy in the UK and Germany. Education and Training, 59(7-8), 720-739. https://doi.org/10.1108/ET-01-20160015

Throsby, D. (2001). Economics and culture. Cambridge: Cambridge University Press.

Throsby, D. (2010). The Economics of Cultural Policy. Cambridge: Cambridge University Press.

Throsby, D. (2017). The rough journey into arts entrepreneurship: Why arts entrepreneurship education is still in its infancy in the UK and Germany. Education + Training, 59(7), 720-739. https://doi.org/10.1108/ET-01-2016$\underline{0015}$

Toghraee, M. T., \& Monjezi, M. (2017). Introduction to Cultural Entrepreneurship: Cultural Entrepreneurship in Developing Countries. International Review of Management and Marketing, 7(4), 67-73.

Toscher, B. (2020). Get Give Make Live: An Empirical Comparative Study of Motivations for Technology, Youth and Arts Entrepreneurship. Social Enterprise Journal, 16(2), 179-202. https://doi.org/10.1108/SEJ-03-2019-0016

Townley, B.; Gulledge, E. (2015). The Market for Symbolic Goods: Translating Economic and Symbolic Capitals in Creative Industries. In C. Jones, M. Lorenzen, \& J. Sapsed (Eds.), The Oxford Handbook of Creative Industries. (119-135). Oxford: Oxford University Press.

Vogt, S., Bulgacov, Y. L. M., \& Elias, S. R. S. T. A. (2019). Entrepreneurial Learning in Practice: Fragments of Aesthetic-Sensible Knowledge in Entrepreneurs' Life Trajectory. Paper presented at the 4th Annual Entrepreneurship as Practice Conference. Nantes, France. 
Warren, L., \& Anderson, A. (2009). Playing the fool? An aesthetic performance of an entrepreneurial identity. In D. Hjorth \& C. Steyaert (Eds.), The politics and aesthetics of entrepreneurship (148-161). Cheltenham: Edward Elgar.

Warren, S. (2008). Empirical challenges in organizational aesthetics research: Towards a sensual methodology. Organization Studies, 29(4), 559-580. https://doi.org/10.1177/0170840607083104

Wennes, G. (2009). Art, Creativity and the New. In Koivunen, N., Rehn, A. (Eds). Creativity and the Contemporary Economy. Copenhagen: Copenhagen Business School.

Werthes, D., Mauer, R., \& Brettel, M. (2017). Cultural and creative entrepreneurs: understanding the role of entrepreneurial identity. International Journal of Entrepreneurial Behavior \& Research, 24(1), 290-314. https://doi.org/10.1108/IJEBR-07-2016-0215

White, J. C. (2015). Toward a Theory of Arts Entrepreneurship. Jaee. Journal of Arts Entrepreneurship Education, 1(1), 55-78. https://doi.org/10.46776/jaee.v1

White, J. C. (2017). Analyzing Entrepreneurship in the U.S. Arts Sector: Identifying Arts Entrepreneurs' Demographics and Shared Characteristics. Artivate: A Journal of Entrepreneurship in the Arts, 6(1), 8-32.

Willerding, I. A. V. (2015). Arquétipo para o Compartilhamento do Conhecimento à Luz da Estética Organizacional e da Gestão Empreendedora. Tese de Doutorado. Universidade Federal de Santa Catarina. Brasil. Disponível: https://repositorio.ufsc.br/xmlui/handle/123456789/169636

Wilson, N. C., \& Stokes, D. (2002). Cultural entrepreneurs and creating exchange. Journal of Research in Marketing Entrepreneurship, 4(2), 37-52. https://doi.org/10.1108/14715200280001465

Wilson, N. C., \& Stokes, D. (2004). Laments and serenades: relationship marketing and legitimation strategies for the cultural entrepreneur. Qualitative Market Research: An International Journal, 7(3), 218-227. https://doi.org/10.1108/13522750410540227

Wilson, N. C., \& Stokes, D. (2005). Managing creativity and innovation: The challenge for cultural entrepreneurs. Journal of Small Business and Enterprise Development, 12(3), 366-378. https://doi.org/10.1108/14626000510612286

Wood Jr, T., \& Csillag, P. (2001). Estética organizacional. Organizações \& Sociedade, 8(21), 35-44. https://doi.org/10.1590/o\&s.v8i21.10571

Yoon, D. (2017). The regional-innovation cluster policy for R\&D efficiency and the creative economy with focus on Daedeok Innopolis. Journal of Science and Technology Policy Management, 8(2), 206-226. https://doi.org/10.1108/JSTPM-09-2016-0025

Zelizer, V. (2005). Culture and Consumption. In Neil J.S.; Swedberg, R (Eds.). The Handbook of Economic Sociology. ( $2^{\mathrm{a}}$ ed.). (331-354). Princeton: Princenton University Press. 